\title{
Lorentz-violating inflationary magnetogenesis
}

\author{
Leonardo Campanelli ${ }^{\mathrm{a}}$ \\ Dipartimento di Fisica, Università di Bari, 70126 Bari, Italy
}

Received: 20 May 2015 / Accepted: 8 June 2015 / Published online: 23 June 2015

(C) The Author(s) 2015. This article is published with open access at Springerlink.com

\begin{abstract}
A non-conformally invariant coupling between the inflaton and the photon in the minimal Lorentz-violating standard model extension is analyzed. For specific forms of the Lorentz-violating background tensor, the strongcoupling and back-reaction problems of magnetogenesis in de Sitter inflation with scale $\sim 10^{16} \mathrm{GeV}$ are evaded, the electromagnetic-induced primordial spectra of (Gaussian and non-Gaussian) scalar and tensor curvature perturbations are compatible with cosmic microwave background observations, and the inflation-produced magnetic field directly accounts for cosmic magnetic fields.
\end{abstract}

\section{Introduction}

Coherent magnetic fields as strong as $B \sim 10^{-6} \mathrm{G}$ have been detected in any type of galaxies and in galaxy clusters (for reviews on cosmic magnetic fields, see [1-10]). Their origin is still an open issue and is puzzling to the point that "cosmic magnetism" should be considered one of the biggest mysteries in cosmology.

Nowadays, what it is clear enough is that seed magnetic fields present prior to galaxy formation can be amplified by protogalaxy collapse and magnetohydrodynamic turbulence effects and then, at least in principle, they can reproduce the properties of presently observed galactic fields.

Indeed, it has been recently pointed out [11] (see also references therein) that a small-scale dynamo could exponentially amplify small-scale seed magnetic fields during the process of galactic disk formation. Successively, differential rotation of the newly formed galactic disk would order the chaotic field resulting from the small-scale dynamo in such a way to reproduce the main features of the observed galactic magnetic fields. This mechanism would explain galactic magnetism if a sufficiently strong seed field is present prior

a e-mail: leonardo.campanelli@ba.infn.it to galaxy formation but leave substantially unanswered the question of the presence of strong magnetic fields in clusters of galaxies.

A plethora of mechanism acting in the early Universe have been proposed to produce seed fields since Fermi's proposal of the existence of cosmic magnetic fields back in 1949 [12].

Promising candidates are those mechanism operating during inflation since inflation-generated fields can be correlated on superhorizon scales, and then their comoving correlation length can be as large as the galactic one. If magnetic fields are created after inflation, instead, their correlation length cannot exceed the dimension of the horizon at the time of generation, so that they are correlated on scales generally much smaller than the characteristic scale of the observed cosmic fields.

Since standard Maxwell electromagnetism in a FriedmannRobertson-Walker universe is invariant under conformal transformations, magnetic fields cannot be generated during inflation, as a consequence of the well-known "Parker theorem" $[13,14]$. For this reason, all inflationary models proposed in the literature repose on the breaking of conformal invariance of (standard) electrodynamics.

Turner and Widrow [15] analyzed the consequences of adding, to the Maxwell Lagrangian, nonstandard conformalbreaking gravitational couplings of the photon.

Ratra [16], instead, introduced a nonstandard conformalbreaking coupling between the scalar field $\phi$ responsible for inflation (the inflaton) and the electromagnetic field.

After these two seminal papers on the generation of largescale magnetic fields at inflation, many other mechanisms have been proposed, most of which introduces nonstandard photon couplings to break conformal invariance (see, e.g., [17-59]).

There are, however, three mechanisms proposed in the literature that work without resorting to nonstandard physics.

Dolgov [60] argued that the well-known conformal anomaly in quantum field theory in curved spacetime induces a breaking of conformal invariance of standard electrody- 
namics, which in turn stimulates the generation of strong, large-scale magnetic field at inflation.

Barrow and Tsagas [61,62] (see, also, $[63,64]$ and $[65$, 66]) showed that, within the framework of conventional electromagnetism, astrophysically interesting magnetic fields can be generated if one assumes, contrarily to what previously assumed in the literature of cosmic magnetic fields, that the spatial curvature of the Universe is nonzero and compatible with astrophysical observations.

The author pointed out in [67] (see, also, [68-72]) that the process of renormalization of inflationary quantum magnetic fluctuations naturally breaks conformal invariance giving, as a result, a strong, scale-independent today magnetic field.

Recently, however, a potential problem for inflationary mechanisms of magnetogenesis has been pinpointed by Demozzi, Mukhanov, and Rubinstein [73], and it is now known as the "strong-coupling problem". The problem consists in the fact that the full electrodynamics theory, including both the nonstandard couplings of the photon with other fields (as the inflaton) and the standard one with conserved external currents, must always be in a weak-coupling regime, in order to have reliable results. This problem, when combined with the so-called "back-reaction problem", which appears in the theory when the inflation-produced electromagnetic field appreciably back-reacts on the inflationary dynamics, excludes all the models of inflationary magnetogenesis based on nonstandard physics.

After this work [73], only three scenarios for inflationary magnetogenesis have been suggested in which both the strong-coupling and the back-reaction problems are avoided. (Such problems are successfully evaded also in a magnetogenesis model proposed by Membiela [74]. In such a model, however, the background cosmology is given by a nonstandard bouncing cosmological model instead of standard inflation.)

Ferreira et al. [75,76] considered a magnetogenesis scenario à la Ratra where the inflaton is kinetically coupled to the photon and where a low scale inflation is followed by a prolonged reheating phase dominated by a stiff fluid.

Caprini and Sorbo [77] proposed a generalization; a Ratralike model, where both a kinetic and an axion-like coupling are present.

Very interesting is the scenario recently proposed by Tasinato [78]. A (nonstandard) derivative interaction between fermion fields (which give rise to the external currents) and a scalar field (which is kinetically coupled to the photon and amplifies electromagnetic vacuum fluctuations) "renormalizes" the electric charge during inflation in such a way that the theory is always in the weak-coupling regime. (For possible problems that could arise in this scenario, see [79].)

Beside the strong-coupling and the back-reaction problems, there is another possible problem, hereinafter referred to as the "curvature perturbation problem", first pointed out by Barnaby et al. [80]. It consists in the fact that inflationary electromagnetic fields generate both scalar (Gaussian and non-Gaussian) and tensor curvature perturbations that could be in conflict with recent observations of cosmic microwave background (CMB) anisotropies.

In this paper, we discuss a generalization of the Ratra model where the inflaton $\phi$ is kinetically coupled to the photon through a Lorentz-violating coupling of the form

$f(\phi)\left(\mathcal{L}_{\mathrm{M}}+\mathcal{L}_{\mathrm{LV}}\right)$,

where $f$ is a generic positive-defined function, $\mathcal{L}_{\mathrm{M}}$ is the standard Maxwell Lagrangian, while $\mathcal{L}_{\mathrm{LV}}$ contains all Lorentzviolating terms that involve the photon field and that are implemented by external background tensors.

The motivation behind the investigation of possible effects of Lorentz-violation in inflationary magnetogenesis is that in some theories of quantum gravity, such as loop quantum gravity [81] and string theory [82], the breakdown of Lorentz symmetry is expected to take place around the Planck scale, and so before the beginning of inflation.

Working in the weak-coupling regime, we will show that strong, scaling-invariant, magnetic fields can be created without back-reacting on the inflationary dynamics and without generating curvature perturbations in conflict with $\mathrm{CMB}$ results. This is possible if the external tensors, which represent new degrees of freedom with respect to the Ratra model, assume specific (fine-tuned) forms that assure that the electric part of the electromagnetic energy-momentum tensor (which would give rise to the the three aforementioned problems) is vanishing during inflation.

The paper is organized as follows. In Sect. 2, we discuss the characteristics (intensity and correlation length) that a comoving cosmic magnetic field must have in order to explain the magnetic fields detected in galaxies and clusters of galaxies. In Sect. 3, we briefly review, in the context of the Ratra-like model, the strong-coupling and backreaction problems in inflationary magnetogenesis. In Sects. 4 and 5, we introduce and quantize our model of magnetogenesis based on Lorentz-violating couplings between the inflaton and the photon. In Sect. 6, we derive the conditions under which the inflation-produced electromagnetic field does not appreciably back-react on the inflationary dynamics. In Sect. 7, we evolve the produced magnetic field from the end of inflation until today. In Sect. 8, we discuss additional constraints that could be eventually imposed on the inflationproduced electromagnetic energy-momentum tensor. In Sect. 9, we calculate the spectrum, bispectrum, and trispectrum of the scalar curvature perturbations and estimate the spectrum of the tensor modes generated by the electromagnetic field, and compare them to the current bounds derived by the Planck mission. In Sect. 10, we discuss our results. Finally, in Sect. 11, we draw our conclusions. 


\section{Seed magnetic fields}

Magnetic fields have been detected in all types of galaxies with intensities of order $\mu \mathrm{G}$. Galaxies at high redshift (still in the process of being formed) and irregular galaxies do not possess structured magnetic fields, while magnetic fields in fully formed galaxies, such as spiral or barred galaxies, typically trace the large-scale structure of galaxies $[1,5]$.

These observations could be explained if a sufficiently intense large-scale magnetic field were present prior to galaxy formation. In this case, and due to the high conductivity of the protogalactic plasma, the magnetic field would remain frozen in the plasma and its final spatial configuration would reflect that of the galaxy. This rearrangement of the structure (not amplification) of the magnetic field could easily be realized by a galactic dynamo action, whose efficiency in reorganizing the primordial field is subjected to only this condition: that the comoving magnetic correlation length be greater than about $100 \mathrm{pc}$ [1]. Moreover, due to the Alfvén theorem (see, e.g., [3]), a frozen-in magnetic field is amplified by a factor of $\left[\rho_{\mathrm{gal}} / \rho_{\mathrm{m}}(t)\right]^{2 / 3}$ and its correlation decreased by $\left[\rho_{\mathrm{gal}} / \rho_{\mathrm{m}}(t)\right]^{1 / 3}$ during protogalactic collapse [15]. Here, $\rho_{\text {gal }}$ and $\rho_{\mathrm{m}}(z) \propto(1+z)^{3}$ are, respectively, the galactic and cosmic matter densities, and for typical galaxies $\rho_{\mathrm{gal}} / \rho_{\mathrm{m}}\left(t_{0}\right) \sim 10^{6}$ at the present cosmic time $t_{0} \simeq 4 \times 10^{17} \mathrm{~s}$. Therefore, a comoving seed field $B_{0} \sim 10^{-10}\left[\left(1+z_{\mathrm{gal}}\right) /\left(1+z_{\mathrm{ta}}\right)\right]^{2} \mathrm{G}$, correlated on a comoving scale greater than $\lambda_{\mathrm{B}} \sim 10\left[\left(1+z_{\mathrm{ta}}\right) /\left(1+z_{\mathrm{gal}}\right)\right] \mathrm{kpc}$, could explain the galactic magnetism. The redshift-dependent factors come from the fact that between the turn-around redshift $z_{\text {ta }}$ (when the protogalactic collapse begins) and the galaxy redshift $z_{\mathrm{gal}}$, a frozen-in primordial magnetic field is decoupled from the Hubble flow and does not evolve adiabatically. Typically, $z_{\mathrm{ta}} \sim$ few tens, while $z_{\mathrm{gal}}$ ranges from 0 to few [2].

The observation of galaxy clusters reveals the presence of intracluster large-scale-correlated $\mu \mathrm{G}$ magnetic fields. The intensity of such fields rise to tens of $\mu \mathrm{G}$ in the cluster cores, but this can be probably ascribed to fast-acting dynamo mechanisms due to cluster cooling flows [1].

Numerical simulations [83] of cluster formation starting at redshift $z_{\text {ta }}=15$ have shown that a few $\times 10^{-10} \mathrm{G}$ seed field is processed by magnetohydrodynamic effects in such a way to reproduce the observed magnetic Faraday rotation maps of clusters at low redshifts $\left(z_{\mathrm{cl}} \simeq 0\right)$. It has also be found that the initial magnetic field correlation properties are inessential to the final result, although the scale of the initial magnetic field fluctuations was limited by the resolution length of order $100 \mathrm{kpc}$ [84]. The overall amplification of a factor few $\times 10^{3}$ is explained as a Alfvén frozen-flux effect of $\left[\rho_{\mathrm{cl}} / \rho_{\mathrm{m}}\left(t_{0}\right)\right]^{2 / 3} \sim 10^{2}$ during cluster collapse [since, typically, $\rho_{\mathrm{cl}} / \rho_{\mathrm{m}}\left(t_{0}\right) \sim 10^{3}$ ], plus an amplification of a factor of few tens, probably due to a Kelvin-Helmholtz instability of the intracluster plasma flows [83]. Therefore, a comoving seed field $B_{0} \sim 10^{-10}\left(1+z_{\mathrm{ta}}\right)^{-2} \mathrm{G}$, correlated on a comoving scale greater than $\lambda_{\mathrm{B}} \sim 100\left(1+z_{\mathrm{ta}}\right) \mathrm{kpc}$, could explain cluster magnetic fields.

Roughly speaking, then, in order to explain both galactic magnetism and galaxy cluster magnetic fields, it suffices to have a comoving seed magnetic field such that [67]

$10^{-13} \mathrm{G} \lesssim B_{0} \lesssim$ few $\times 10^{-12} \mathrm{G}$,

$\lambda_{\mathrm{B}} \gtrsim$ few $\times$ Mpc.

Limits on primordial magnetic fields. If cosmic magnetic fields are relics from inflation, they could modify the standard evolution of the universe in the radiation and matter eras. However, this is not the case, since the present limits on primordial magnetic fields do not exclude the existence of large-scale magnetic fields as strong as those in Eq. (2). Indeed, the most significant limits on large-scale cosmic magnetic fields come from big bang nucleosynthesis analyses, $B_{0} \lesssim 1 \times 10^{-6} \mathrm{G}$ [85-87], data on large-scale structures, $B_{0} \lesssim$ few $\times 10^{-9} \mathrm{G}[87,88]$, studies of CMB radiation, $B_{0} \lesssim$ few $\times 10^{-9} \mathrm{G}[87,89-93]$, studies of the ionization history of our Universe, $B_{0} \lesssim 10^{-9} \mathrm{G}$ [94], Faraday rotation maps of distant quasars, $B_{0} \lesssim 10^{-11} \mathrm{G}[95,96]$, and blazar observations, $B_{0} \gtrsim 7 \times 10^{-14} \mathrm{G}$ [97-99], where the last lower limit refers to the less conservative bound from the blazar 1ES 0229+200 [99]. It is interesting to observe that the upper limit from Faraday rotation maps and the lower limit from blazar observations are a just few times outside the interval of $B_{0}$ in Eq. (2). Narrowing the above limits could then eventually reveal the primordial nature of cosmic magnetic fields.

\section{Strong coupling and back-reaction in inflationary magnetogenesis}

Let us now discuss, in some detail, two requirements that have to be imposed on any magnetogenesis mechanism operating during inflation. We focus our attention to the "standard" kinetically coupled scenario for magnetogenesis, where the inflaton field $\phi$ is coupled to the standard kinetic Maxwell term via a generic coupling $f(\phi)$. This represents an extended version of the model proposed by Ratra [16], where $f(\phi) \propto e^{\alpha \phi}$, with $\alpha$ being a constant.

The first requirement, that the full theory, namely when including conserved external currents, must be in a weakcoupling regime, has been discussed only recently in [73].

The second requirement, namely that the inflationproduced electromagnetic field must not appreciably backreact on the inflationary dynamics, has been instead first discussed by Ratra [16], but it was ignored in the seminal paper [15] by Turner and Widrow on inflationary magnetogenesis. 


\subsection{Strong coupling}

Let us consider the action for the electromagnetic field $A_{\mu}$,

$S_{\text {em }}=\int \mathrm{d}^{4} x \sqrt{-g} \mathcal{L}_{\mathrm{em}}$,

where $g$ is the determinant of the metric tensor $g_{\mu \nu}$, and $\mathcal{L}_{\mathrm{em}}$ the electromagnetic Lagrangian density, and let us assume that the electromagnetic field is coupled to the (homogeneous) inflaton field $\phi$ through a general coupling of the form

$\mathcal{L}_{\text {em }}=f(\phi) \mathcal{L}_{\mathrm{M}}+\mathcal{L}_{\text {int }}$.

Here, $f(\phi)$ is a generic, positive-defined function of the inflaton, $\mathcal{L}_{\mathrm{M}}=-\frac{1}{4} F_{\mu \nu} F^{\mu \nu}$ is the standard free Maxwell Lagrangian density, with $F_{\mu \nu}=\partial_{\mu} A_{\nu}-\partial_{\nu} A_{\mu}$, while $\mathcal{L}_{\text {int }}=$ $j^{\mu} A_{\mu}$ is the standard interaction term with conserved external current. If, for the sake of simplicity, we assume that $j_{\mu}$ is provided just by a charged massless fermion fields $\psi$, we than have $j^{\mu}=e \bar{\psi} \gamma^{\mu} \psi$, where $e$ is the electric charge, and $\gamma^{\mu}$ are the Dirac matrices in curved spacetime. The latter are related to the standard Dirac matrices in Minkowski spacetime through $\gamma^{\mu}=e_{a}^{\mu} \gamma^{a}$, with $e_{a}^{\mu}$ being the vierbein [13]. ${ }^{1}$

(In this paper, indices in Minkowski spacetime are indicated with the first letters of the Latin alphabet and run from 0 to 3. Indices in curved spacetimes are indicated with Greek letters and run from 0 to 3 . Latin indices from the middle of the alphabet run from 1 to 3 and indicates spatial components of a given tensor.)

Re-writing the Lagrangian density (5) as

$\mathcal{L}_{\mathrm{em}}=f(\phi)\left(\mathcal{L}_{\mathrm{M}}+\frac{e}{f(\phi)} \bar{\psi} \gamma^{\mu} \psi\right)$

we see that the quantity $e / f(\phi)$ plays the role of an effective, time-dependent electric charge. The case $f(\phi) \ll 1$ would then correspond to a strong coupling between the fermion and the electromagnetic fields, and the theory would be in a (unmanageable) strong-coupling regime, as first pointed out in [73]. For this reason, we assume that $f(\phi) \gtrsim 1$ during inflation and, obviously, $f(\phi) \simeq 1$ at the end of inflation in order to recover the standard electrodynamics. Accordingly, we will consistently neglect, in the following, the interaction term $\mathcal{L}_{\text {int }}$ in Eq. (5).

\subsection{Backreaction}

It is tacitly assumed in the literature that inflationary magnetogenesis takes place in a fixed curved spacetime background. Therefore, we must consistently check that the vacuum expectation value (VEV) of the electromagnetic energy-

1 The vierbein satisfies the condition $e_{a}^{\mu} e_{b \mu}=\eta_{a b}$, and is such that $g_{\mu \nu}=e_{\mu}^{a} e_{\nu}^{b} \eta_{a b}$, where $\eta_{a b}$ is the metric tensor in Minkowski spacetime. momentum tensor is always negligible with respect to the energy-momentum tensor of the inflaton.

The electromagnetic energy-momentum tensor can be found by varying the action with respect to the metric tensor,

$\left(T_{\mathrm{em}}\right)_{\mu \nu}=\frac{2}{\sqrt{-g}} \frac{\delta S_{\mathrm{em}}}{\delta g^{\mu \nu}}$.

We obtain $\left(T_{\mathrm{em}}\right)_{\mu \nu}=f(\phi)\left(T_{\mathrm{M}}\right)_{\mu \nu}$, where $\left(T_{\mathrm{M}}\right)_{\mu \nu}=$ $F_{\alpha \mu} F_{\nu}{ }^{\alpha}+\frac{1}{4} F_{\alpha \beta} F^{\alpha \beta} g_{\mu \nu}$ is the standard Maxwell energymomentum tensor.

Let us restrict our analysis to the case of a spatially flat, Friedmann-Robertson-Walker universe, described by the line element

$\mathrm{d} s^{2}=a^{2}\left(\mathrm{~d} \eta^{2}-\mathrm{d} \mathbf{x}^{2}\right)$,

where $\eta$ is the conformal time and $a(\eta)$ is the expansion parameter [the latter is normalized to unity at the present conformal time $\left.\eta_{0}, a\left(\eta_{0}\right)=1\right]$. Moreover, we assume, for the sake of simplicity, that inflation is described by a de Sitter phase. In this case, the conformal time is inversely proportional to the expansion parameter, $\eta=-1 / H a$, and the Hubble parameter $H$ is a constant. Moreover, the energymomentum tensor of the inflaton is $\left(T_{\nu}^{\mu}\right)_{\text {inf }}=M^{4} \delta_{\nu}^{\mu}$, where $\delta_{v}^{\mu}$ is the Kronecker delta. Here, $M$ is the scale of inflation, related to the energy density of inflation, $\rho_{\text {inf }}$, through $M^{4}=\rho_{\text {inf }}=3 H^{2} /(8 \pi G)$, where $G=1 / m_{\mathrm{Pl}}^{2}$ is the Newton constant and $m_{\mathrm{Pl}} \sim 10^{19} \mathrm{GeV}$ is the Planck mass.

Since both the background spacetime and the coupling function $f(\phi)$ are homogeneous and isotropic, the VEV of the electromagnetic energy-momentum tensor takes on the simple form

$\left\langle\left(T_{\mathrm{em}}\right)_{v}^{\mu}\right\rangle=\rho_{\mathrm{em}} \operatorname{diag}(1,-1 / 3,-1 / 3,-1 / 3)$,

where $\rho_{\mathrm{em}}=\left\langle\left(T_{\mathrm{em}}\right)_{0}^{0}\right\rangle$ is the VEV of the electromagnetic energy density. (For the quantization of the theory and a formal definition of the vacuum, see Sect. 5.) Consequently, the condition that the electromagnetic back-reaction on inflation is negligible can be expressed as $\rho_{\mathrm{em}} \ll \rho_{\text {inf }}$. The electromagnetic energy density is made up of an electric contribution and a magnetic part,

$\rho_{\mathrm{em}}=f(\phi)\left(\frac{1}{2}\left\langle\mathbf{E}^{2}\right\rangle+\frac{1}{2}\left\langle\mathbf{B}^{2}\right\rangle\right)$,

where the electric and magnetic fields are defined as usual as $a^{2} \mathbf{E}=-\dot{\mathbf{A}}$ and $a^{2} \mathbf{B}=\nabla \times \mathbf{A}$, with $A_{\mu}=(0, \mathbf{A})$. Here, and in the following, we work the Coulomb gauge, $A_{0}=\partial_{i} A_{i}=$ 0 , we denote the differentiation with respect to the conformal time with a dot, and we use the symbol $\nabla$ for indicating the nabla operator in comoving coordinates.

The two-point correlators $\left\langle\mathbf{E}^{2}\right\rangle$ and $\left\langle\mathbf{B}^{2}\right\rangle$ are formally infinite due to the ultraviolet divergence of the corresponding spectra. This kind of divergence, typical in quantum theory in curved spacetime, can be cured by the standard techniques 
of renormalization, such as adiabatic renormalization. Nevertheless, we are principally interested in large-scale electromagnetic modes which are outside the horizon. These modes, which are expected to behave classically, belong to the nondivergent, infrared part of the spectra. Therefore, it is convenient to work in Fourier space and introduce the so-called electric and magnetic power spectra, $\mathcal{P}_{\mathrm{E}}(k, \eta)$ and $\mathcal{P}_{\mathrm{B}}(k, \eta)$, through

$$
\left\langle\mathbf{E}^{2}\right\rangle=\int_{0}^{\infty} \frac{\mathrm{d} k}{k} \mathcal{P}_{\mathrm{E}}(k), \quad\left\langle\mathbf{B}^{2}\right\rangle=\int_{0}^{\infty} \frac{\mathrm{d} k}{k} \mathcal{P}_{\mathrm{B}}(k) .
$$

The electromagnetic energy density stored on the mode $k$ is then

$\rho_{\mathrm{em}}(k)=f(\phi)\left[\frac{1}{2} \mathcal{P}_{\mathrm{E}}(k)+\frac{1}{2} \mathcal{P}_{\mathrm{B}}(k)\right]$,

where $\rho_{\mathrm{em}}(k, \eta)$ is the electromagnetic energy spectrum defined by $\rho_{\mathrm{em}}=\int_{0}^{\infty} \frac{\mathrm{d} k}{k} \rho_{\mathrm{em}}(k)$.

The condition that the electromagnetic back-reaction on inflation is negligible can then be defined, mode-by-mode, by

$$
\rho_{\mathrm{em}}(k) \lesssim \rho_{\mathrm{inf}} \sim 10^{10}\left(\frac{M}{10^{16} \mathrm{GeV}}\right)^{-4} H^{4} .
$$

A particularly interesting class of models is that for which the coupling function $f(\phi)$ scales in time as $f(\phi) \propto \eta^{6}$. This gives a scale-invariant magnetic spectrum, to wit $\mathcal{P}_{\mathrm{B}}(k)$ independent on $k$. The attractive figure of this model resides in the fact that, as firstly pointed out in [100], all the existing constraints on cosmic magnetic fields do not strongly peak over a specific range of either small or large scales. Hence, a scaling-invariant magnetic field can satisfy, in a "natural way", all the current experimental bounds, included the one in Eq. (3). For the scaling-invariant case, the electric and magnetic spectra are, roughly speaking,

$\mathcal{P}_{\mathrm{E}}(k) \sim \frac{\mathcal{P}_{B}(k)}{(-k \eta)^{2}}, \quad \mathcal{P}_{\mathrm{B}}(k) \sim \frac{H^{4}}{f(\phi)}$.

On superhorizon scales $(-k \eta \ll 1)$, then, the dominant contribution to the electromagnetic energy-momentum tensor is provided by the electric part. Its maximum value is attained at the end of inflation, $\eta=\eta_{\text {end }}$. Therefore, back-reaction on inflation is negligible on scales $\lambda=1 / k$ such that

$\lambda \lesssim \lambda_{\max }=10^{-16}\left(\frac{M}{10^{16} \mathrm{GeV}}\right)^{-3} \mathrm{Mpc}$,

where we used the fact that $-k \eta_{\text {end }} \sim 10^{-22}(\mathrm{Mpc} / \lambda)$ $\left(10^{16} \mathrm{GeV} / M\right)$.

To simplify the analysis, we have considered here the case of instantaneous reheating, to wit, we have assumed that after inflation the Universe entered directly in the radiation dominated era. Needless to say, the electromagnetic back-reaction on inflation has to be negligible on all observable scales. This, in turns, means that $\lambda_{\max }$ has to be greater than the present horizon scale, $H_{0}^{-1} \simeq 4000 \mathrm{Mpc}$, where $H_{0}$ is the Hubble constant. Accordingly, the scale of inflation has to be below $10^{9} \mathrm{GeV}$. Such a low scale seems to be incompatible with recent results on the detection of inflation-produced gravitational waves, which require a scale of inflation around $10^{16} \mathrm{GeV}$ [101]. However, even assuming a scale as low as $M \sim 10^{9} \mathrm{GeV}$, the amplitude of the inflation-produced magnetic field would be today too small to directly explain cosmic magnetism. Indeed, the actual magnetic field for the scalinginvariant case is

$$
B_{0} \sim 10^{-12}\left(\frac{M}{10^{16} \mathrm{GeV}}\right)^{2} \mathrm{G},
$$

and it assumes the extremely low value $B_{0} \sim 10^{-26} \mathrm{G}$ for $M \sim 10^{9} \mathrm{GeV}$.

\section{Lorentz-violating couplings}

The arguments in Sect. 3 clearly show that the generation of (scaling-invariant) magnetic fields during (de Sitter) inflation, able to directly explain cosmic magnetization, is problematic due to their strong back-reaction effects. The validity of this sort of no-go theorem for inflationary magnetogenesis, however, is not general, but it is restricted to the specific model described by the Lagrangian density (5). This leaves open the possibility to explore different couplings between the inflaton and the photon that may eventually generate, in a self-consistent way, cosmic magnetic fields.

In the following, we investigate one such a possibility, by looking at a possible new interaction of the inflaton with the electromagnetic field, this time in the context of the Lorentzviolating extension of the standard model of particle physics (for other mechanisms of cosmic magnetic fields at inflation reposing on the violation of Lorentz symmetry see [102111]).

\subsection{Lagrangian}

The photon sector of the minimal Lorentz-violating standard model extension (SME) is described by the action

$S_{\gamma}=\int \mathrm{d}^{4} x \sqrt{-g}\left(\mathcal{L}_{\mathrm{M}}+\mathcal{L}_{\mathrm{LV}}\right)$,

where $\mathcal{L}_{\mathrm{M}}$ is the Lorentz- and CPT-invariant Maxwell Lagrangian density, while

$\mathcal{L}_{\mathrm{LV}}=\mathcal{L}_{\mathrm{CPT}-\text { even }}+\mathcal{L}_{\mathrm{CPT}-\text { odd }}$

contains all Lorentz-violating terms that involve the photon field. They can be separated into two parts, $\mathcal{L}_{\mathrm{CPT}-\text { even }}$ and $\mathcal{L}_{\mathrm{CPT}-\text { odd }}$, with the former preserving and the latter violating CPT symmetry, respectively. 
In Minkowski spacetime, Lorentz violation is achieved by coupling the electromagnetic field to rank- $n$, constant spacetime tensors $k_{a_{1} a_{2} \ldots a_{n}}$, known as external or background tensors. The passage from Minkowski to a general curved spacetime is obtained via the vierbein $e_{\mu}^{a}, k_{\mu_{1} \mu_{2} \ldots \mu_{n}}=$ $e_{\mu_{1}}^{a_{1}} e_{\mu_{2}}^{a_{2}} \ldots e_{\mu_{n}}^{a_{n}} k_{a_{1} a_{2} \ldots a_{n}}$ [112]. In this passage, however, the external tensors acquire a spacetime dependence and then cease to be constant. This is due to the fact that the vierbein $e_{\mu}^{a}(x)$ is, generally, a function of the spacetime position $x$.

In the photon sector, the most general renormalizable Lagrangian density contains only three Lorentz-violating terms,

$$
\begin{aligned}
& \mathcal{L}_{\mathrm{CPT}-\text { even }}=-\frac{1}{4}\left(k_{F}\right)_{\mu \nu \alpha \beta} F^{\mu \nu} F^{\alpha \beta}, \\
& \mathcal{L}_{\mathrm{CPT}-\text { odd }}=\frac{1}{2}\left(k_{A F}\right)_{\mu} A_{\nu} \widetilde{F}^{\mu \nu}-\left(k_{A}\right)_{\mu} A^{\mu},
\end{aligned}
$$

where $\left(k_{F}\right)_{\mu \nu \alpha \beta},\left(k_{A F}\right)_{\mu}$, and $\left(k_{A}\right)_{\mu}$, are background tensors. Their components are arbitrary real spacetime functions and are known as coefficients for Lorentz violation. Although the presence of the external tensors may indicate an explicit breaking of Lorentz violation, the form of the Lagrangian terms (19) and (20) is completely general and independent of the origin of the Lorentz violation. Indeed, these terms would have the same form in the case where Lorentz violation were spontaneous, deriving, for example, from the fact that the external tensor $k_{\mu_{1} \mu_{2} \ldots \mu_{n}}$ are vacuum expectation values of corresponding field operators $\mathcal{K}_{\mu_{1} \mu_{2} \ldots \mu_{n}}$,

$k_{\mu_{1} \mu_{2} \ldots \mu_{n}}=\left\langle 0\left|\mathcal{K}_{\mu_{1} \mu_{2} \ldots \mu_{n}}\right| 0\right\rangle$.

We now generalize the coupling in Eq. (5) by assuming that the inflaton is coupled to the photon field, via the generic function $f(\phi)$, to both the standard photon kinetic term, $\mathcal{L}_{\mathrm{M}}$, and the Lorentz-violating term, $\mathcal{L}_{\mathrm{LV}}$,

$\mathcal{L}_{\mathrm{em}}=f(\phi)\left(\mathcal{L}_{\mathrm{M}}+\mathcal{L}_{\mathrm{LV}}\right)$.

For the sake of simplicity, we consider only the CPT-even terms in the Lagrangian density (18). The electromagnetic Lagrangian density then reads

$\mathcal{L}_{\mathrm{em}}=f(\phi) \mathcal{L}_{\mathrm{MK}}$

where

$\mathcal{L}_{\mathrm{MK}}=\mathcal{L}_{\mathrm{M}}-\frac{1}{4}\left(k_{F}\right)_{\mu \nu \alpha \beta} F^{\mu \nu} F^{\alpha \beta}$

is referred to as the Maxwell-Kostelecký Lagrangian density. The dimensionless rank-4 background tensor $\left(k_{F}\right)_{\mu \nu \alpha \beta}$ is antisymmetric on the first two and last two indices, and it is symmetric for the interchange of the first and last pair of indices. These symmetries reduce the number of independent components of $\left(k_{F}\right)_{\mu \nu \alpha \beta}$ to 21. It is useful to decompose $\left(k_{F}\right)_{\mu \nu \alpha \beta}$ into irreducible multiplets [112], $21=1_{a}+1_{s}+9_{s}+10_{s}$, where $1_{a}$ represents an antisymmetric singlet (pseudoscalar), $1_{s}$ a symmetric singlet (scalar), $9_{s}$ a symmetric traceless rank-2 tensor, and $10_{s}$ a rank-4 tensor possessing the same symmetries of $\left(k_{F}\right)_{\mu \nu \alpha \beta}$ and such that any contraction is identically zero.

Let us restrict our analysis to the case where the background tensor in Eq. (24) is constructed from fundamental (not composite) tensors which appear just once in the definition of $\left(k_{F}\right)_{\mu \nu \alpha \beta}$. Excluding the cases where such fundamental tensors are a scalar and/or a pseudoscalar, in which case the resulting theory is Lorentz invariant, we are left with the cases of a fundamental rank-2 symmetric tensor and/or a fundamental rank-4 tensor. In this paper, and for the sake of simplicity, we consider just the case of a fundamental rank2 tensor $\left(k_{F}\right)_{\mu \nu}$, and leave the case of a rank-4 tensor to future investigations. In this specific case, the independent components of $\left(k_{F}\right)_{\mu \nu \alpha \beta}$ reduce to 10 , and they are given by $k_{F}=\left(k_{F}\right)_{\mu}^{\mu}$ and $\left(\widehat{k}_{F}\right)_{\mu \nu}=\left(k_{F}\right)_{\mu \nu}-\frac{1}{4} k_{F} g_{\mu \nu}$, which are, respectively, the trace and the traceless part of the tensor $\left(k_{F}\right)_{\mu \nu}$. Accordingly, the electromagnetic Lorentz-violating Lagrangian density, which describe the coupling between the inflaton and the photon, can be written as

$\mathcal{L}_{\mathrm{em}}=f(\phi)\left(\mathcal{L}_{\mathrm{M}}+\frac{1}{4} \xi_{1} k_{F} F_{\alpha \beta} F^{\alpha \beta}-\xi_{2}\left(\widehat{k}_{F}\right)_{\mu \nu} F^{\mu \alpha} F_{\alpha}^{\nu}\right)$,

where $\xi_{i}$ are real numerical factors. The background tensor $\left(k_{F}\right)_{\mu \nu \alpha \beta}$, when expressed as a function of the fundamental rank-2 tensor $\left(k_{F}\right)_{\mu \nu}$, has the form

$\left(k_{F}\right)_{\mu \nu \alpha \beta}=-\xi_{1} k_{F} g_{\mu[\alpha} g_{\beta] \nu}+4 \xi_{2}\left(\widehat{k}_{F}\right)_{\mu][\alpha} g_{\beta][\nu}$,

where square brackets [...] indicate antisymmetrization of the indices enclosed, e.g., $T_{\mu_{1} \ldots\left[\mu_{i} \mu_{j}\right] \ldots \mu_{n}}=\frac{1}{2}\left(T_{\mu_{1} \ldots \mu_{i} \mu_{j} \ldots \mu_{n}}\right.$ $\left.-T_{\mu_{1} \ldots \mu_{j} \mu_{i} \ldots \mu_{n}}\right)$ and $T_{\left.\mu_{1}\right] \ldots\left[\mu_{n}\right.}=\frac{1}{2}\left(T_{\mu_{1} \ldots \mu_{n}}-T_{\mu_{n} \ldots \mu_{1}}\right)$.

\subsection{Equation of motion}

As in Sect. 3, we restrict our analysis to the case of a spatially flat, Friedmann-Robertson-Walker universe. Since $g_{\mu \nu}=a^{2} \eta_{\mu \nu}$, we can take for the vierbein $e_{\mu}^{b}=a \delta_{\mu}^{b}$, where $\delta_{\mu}^{b}$ is the Kronecker delta. Accordingly, we have $\left(k_{F}\right)_{\mu \nu}=e_{\mu}^{b} e_{\nu}^{c}\left(k_{F}\right)_{c b}=a^{2} \delta_{\mu}^{b} \delta_{\nu}^{c}\left(k_{F}\right)_{c b}$. Let now assume that the background tensor $\left(k_{F}\right)_{a b}$ is homogeneous and isotropic, so that the number of its independent components reduces to 2 . In this case, $\left(k_{F}\right)_{a b}$ can be generally written as $\left(k_{F}\right)_{c b}=\operatorname{diag}\left(\rho_{K}, p_{K}, p_{K}, p_{K}\right)$, where $\rho_{K}$ and $p_{K}$ are two scalar functions which depend only on the conformal time $\eta$. In curved spacetime, then, the background tensor assumes the form

$\left(k_{F}\right)_{v}^{\mu}=\operatorname{diag}\left(\rho_{K},-p_{K},-p_{K},-p_{K}\right)$. 
It is useful, for the following discussion, to introduce the electromagnetic Lagrangian $L_{\mathrm{em}}$ through

$S_{\mathrm{em}}=\int \mathrm{d} \eta L_{\mathrm{em}}$.

Taking into account Eq. (4) and the fact that $\sqrt{-g}=a^{4}$, we have

$L_{\mathrm{em}}=\int \mathrm{d}^{3} x a^{4} \mathcal{L}_{\mathrm{em}}$,

with $\mathcal{L}_{\text {em }}$ given by Eq. (25). Working in the Coulomb gauge, we have

$L_{\mathrm{em}}=\int \mathrm{d}^{3} x\left(\frac{1}{2} \varepsilon \dot{\mathbf{A}}^{2}-\frac{1}{2 \mu}(\nabla \mathbf{A})^{2}\right)$,

where we have defined the time-dependent functions

$\varepsilon=f(\phi)\left[1-\left(\xi_{1}-\xi_{2}\right) \rho_{K}+\left(3 \xi_{1}+\xi_{2}\right) p_{K}\right]$,

$\mu^{-1}=f(\phi)\left[1-\left(\xi_{1}+\xi_{2}\right) \rho_{K}+\left(3 \xi_{1}-\xi_{2}\right) p_{K}\right]$.

Varying the action (30) with respect to $\mathbf{A}$, we find the equation of motion for the vector potential,

$\ddot{\mathbf{A}}+\frac{\dot{\varepsilon}}{\varepsilon} \dot{\mathbf{A}}-\frac{1}{n^{2}} \nabla^{2} \mathbf{A}=0$,

where we have defined $n=\sqrt{\varepsilon \mu}$, and we assume that $\varepsilon$ and $\mu$ are positive-defined quantities.

\subsection{Analogy with continuous media}

It is well known in the literature that there exists an analogy between the photon sector of the minimal SME and the electrodynamics of continuous (or macroscopic) media [113]. In our case, this analogy works as follows. We rewrite the electromagnetic Lagrangian density as

$\mathcal{L}_{\mathrm{em}}=\mathcal{L}_{\mathrm{M}}+\frac{1}{4} \chi^{\mu \nu}{ }_{\alpha \beta} F_{\mu \nu} F^{\alpha \beta}$

where

$$
\begin{aligned}
\chi_{\alpha \beta}^{\mu \nu}= & \frac{1}{2}\left\{1-f(\phi)\left[1-\left(\xi_{1}+\xi_{2}\right) k_{F}\right]\right\} \delta_{\alpha \beta}^{\mu \nu} \\
& -4 \xi_{2} f(\phi) \delta_{[\alpha}^{[\mu}\left(k_{F}\right)_{\beta]}^{\nu]}
\end{aligned}
$$

is the susceptibility tensor, and $\delta_{\alpha \beta}^{\mu \nu}$ the generalized Kronecker delta. Introducing the polarization-magnetization tensor $\mathcal{M}^{\mu \nu}$ as

$\mathcal{M}^{\mu \nu}=\chi_{\alpha \beta}^{\mu \nu} F^{\alpha \beta}$,

the equation of motion is

$\mathcal{D}_{; \mu}^{\mu \nu}=0$,

where

$\mathcal{D}^{\mu v}=F^{\mu v}-\mathcal{M}^{\mu v}$ is the displacement tensor. In the Coulomb gauge, Eq. (37) reduces to $0=0$ for $v=0$, and to Eq. (33) for $v=i$.

Let us introduce the electric and magnetic fields, $a^{2} E_{i}=$ $-F_{0 i}$ and $a^{2} B_{i}=\frac{1}{2} \epsilon_{i j k} F_{j k}$, the displacement and magnetizing fields, $a^{2} D_{i}=-\mathcal{D}_{0 i}$ and $a^{2} H_{i}=\frac{1}{2} \epsilon_{i j k} \mathcal{D}_{j k}$, and the polarization and magnetization fields, $a^{2} P_{i}=\mathcal{M}_{0 i}$ and $a^{2} M_{i}=\frac{1}{2} \epsilon_{i j k} \mathcal{M}_{j k}$.

Equation (38) can then be rewritten, in three-dimensional form, as $\mathbf{D}=\mathbf{E}+\mathbf{P}$ and $\mathbf{H}=\mathbf{B}-\mathbf{M}$, where $\mathbf{X}=$ $\left(X_{1}, X_{2}, X_{3}\right)$, and $\mathbf{X}$ stands for $\mathbf{E}, \mathbf{B}, \mathbf{D}, \mathbf{H}, \mathbf{P}$, or $\mathbf{M}$. Equation (36) gives, instead, $\mathbf{P}=(\varepsilon-1) \mathbf{E}$ and $\mathbf{M}=\left(1-\mu^{-1}\right) \mathbf{B}$, so that

$\mathbf{D}=\varepsilon \mathbf{E}$,

$\mathbf{H}=\mu^{-1} \mathbf{B}$.

The equations connecting the displacement and magnetizing fields to the electric and magnetic fields are known, in the electrodynamic theory of continuous media, as "constitutive relations", and completely determine (together with the boundary conditions) the propagation properties of electromagnetic signals. In particular, Eqs. (39) and (40) describe an isotropic linear medium with electric permittivity $\varepsilon$ and magnetic permeability $\mu$. Accordingly, the evolution in vacuum of electromagnetic fields described by the Lorentz-violating electromagnetic Lagrangian density (34) is formally equivalent to the evolution of electromagnetic fields described by the standard Maxwell theory in a continuous medium with $\varepsilon$ and $\mu$ given by Eqs. (31) and (32). Continuing with the analogy of continuous media, the quantity $n$ defined below Eq. (33) can be interpreted as the refractive index of the medium.

Finally, and for the sake of completeness, we observe that the equation of motion, in terms of the displacement and magnetizing fields, assume the form

$\nabla \cdot\left(a^{2} \mathbf{D}\right)=0, \quad \frac{\partial\left(a^{2} \mathbf{D}\right)}{\partial \eta}=\nabla \times\left(a^{2} \mathbf{H}\right)$,

while the Bianchi identities are

$\nabla \cdot\left(a^{2} \mathbf{B}\right)=0, \quad \frac{\partial\left(a^{2} \mathbf{B}\right)}{\partial \eta}=-\nabla \times\left(a^{2} \mathbf{E}\right)$.

Inserting Eqs. (39) and (40) in the second equation of Eq. (41), we recover Eq. (33).

\section{Quantization}

Let us now quantize the electromagnetic field whose dynamics is described by the Lagrangian (30). 


\subsection{Wronskian condition}

We expand the electromagnetic vector potential as

$\mathbf{A}(\eta, \mathbf{x})=\sum_{\lambda=1}^{2} \int \frac{\mathrm{d}^{3} k}{(2 \pi)^{3} \sqrt{2 k}} \boldsymbol{\varepsilon}_{\mathbf{k}, \lambda} a_{\mathbf{k}, \lambda} A_{k, \lambda}(\eta) \mathrm{e}^{i \mathbf{k x}}+$ H.c.

where $\mathbf{k}$ is the comoving wave number, with $k=|\mathbf{k}|$, and $\boldsymbol{\varepsilon}_{\mathbf{k}, \lambda}$ are the standard circular polarization vectors. ${ }^{2}$

The annihilation and creation operators $a_{\mathbf{k}, \lambda}$ and $a_{\mathbf{k}, \lambda}^{\dagger}$ satisfy the usual commutation relations,

$$
\begin{aligned}
& {\left[a_{\mathbf{k}, \lambda}, a_{\mathbf{k}^{\prime}, \lambda^{\prime}}^{\dagger}\right]=(2 \pi)^{3} \delta_{\lambda \lambda^{\prime}} \delta\left(\mathbf{k}-\mathbf{k}^{\prime}\right),} \\
& {\left[a_{\mathbf{k}, \lambda}, a_{\mathbf{k}^{\prime}, \lambda^{\prime}}\right]=\left[a_{\mathbf{k}, \lambda}^{\dagger}, a_{\mathbf{k}^{\prime}, \lambda^{\prime}}^{\dagger}\right]=0 .}
\end{aligned}
$$

The vacuum state $|0\rangle$ is defined by $a_{\mathbf{k}, \lambda}|0\rangle=0$ for all $\mathbf{k}$ and $\lambda$, and it is normalized as $\langle 0 \mid 0\rangle=1$.

The equation of motion for the two photon polarization states, $A_{k, \lambda}$, is obtained by inserting Eq. (43) in Eq. (33),

$\ddot{A}_{k, \lambda}+\frac{\dot{\varepsilon}}{\varepsilon} \dot{A}_{k, \lambda}+\frac{k^{2}}{n^{2}} A_{k, \lambda}=0$.

In order to have a consistent quantization of the electromagnetic field, the solutions of the above equation must satisfy a normalization condition, known as the Wronskian condition, which can be obtained as follows. Let us introduce the electromagnetic conjugate momentum, $\pi=\left(\pi^{1}, \pi^{2}, \pi^{3}\right)$, as usual as

$\pi=\frac{\delta L_{\mathrm{em}}}{\delta \dot{\mathbf{A}}}=\varepsilon \dot{\mathbf{A}}$,

where in the last equality we used Eq. (30), and let us impose the canonical commutation relation

$\left[A_{i}(\mathbf{x}), \pi^{j}(\mathbf{y})\right]=i \delta_{\perp i}^{j}(\mathbf{x}-\mathbf{y})$,

where

$\delta_{i j}^{\perp}=\int \frac{\mathrm{d}^{3} k}{(2 \pi)^{3}} \mathrm{e}^{i \mathbf{k}(\mathbf{x}-\mathbf{y})}\left(\delta_{i j}-\hat{k}_{i} \hat{k}_{j}\right)$

is the transverse delta function. Inserting Eqs. (43) and (47) in the left hand side of Eq. (48), we find that the latter equation is satisfied only if

$\sum_{\lambda=1}^{2} \boldsymbol{\varepsilon}_{\mathbf{k}, \lambda} \otimes \boldsymbol{\varepsilon}_{\mathbf{k}, \lambda}^{*}\left(W\left[A_{k, \lambda}, A_{k, \lambda}^{*}\right]-\frac{2 i k}{\varepsilon}\right)=0$,

where we used Eqs. (44) and (45). Here,

$W\left[A_{k, \lambda}^{(1)}, A_{k, \lambda}^{(2)}\right]=A_{k, \lambda}^{(1)} \dot{A}_{k, \lambda}^{(2)}-\dot{A}_{k, \lambda}^{(1)} A_{k, \lambda}^{(2)}$

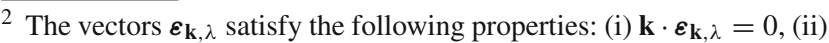
$\boldsymbol{\varepsilon}_{\mathbf{k}, \lambda} \cdot \boldsymbol{\varepsilon}_{\mathbf{k}, \lambda^{\prime}}^{*}=\delta_{\lambda \lambda^{\prime}}$, (iii) $\sum_{\lambda}\left(\boldsymbol{\varepsilon}_{\mathbf{k}, \lambda}\right)_{i}\left(\boldsymbol{\varepsilon}_{\mathbf{k}, \lambda^{\prime}}^{*}\right)_{j}=\delta_{i j}-\hat{k}_{i} \hat{k}_{j}$, (iv) $\boldsymbol{\varepsilon}_{-\mathbf{k}, \lambda}^{*}=$ $-\boldsymbol{\varepsilon}_{\mathbf{k}, \lambda}$, and (v) $i \hat{\mathbf{k}} \times \boldsymbol{\varepsilon}_{\mathbf{k}, \lambda}=(-1)^{\lambda+1} \boldsymbol{\varepsilon}_{\mathbf{k}, \lambda}$, where $\hat{\mathbf{k}}=\mathbf{k} / k$. is the Wronskian of any two independent solutions, $A_{k, \lambda}^{(1)}(\eta)$ and $A_{k, \lambda}^{(2)}(\eta)$, of Eq. (46). Using the Abel identity [114], the above Wronskian can be found explicitly,

$W\left[A_{k, \lambda}^{(1)}(\eta), A_{k, \lambda}^{(2)}(\eta)\right]=W\left[A_{k, \lambda}^{(1)}\left(\eta_{i}\right), A_{k, \lambda}^{(2)}\left(\eta_{i}\right)\right] \frac{\varepsilon\left(\eta_{i}\right)}{\varepsilon(\eta)}$,

where $\eta_{i}$ is an arbitrary time. Accordingly, Eq. (50) can be written as

$$
\sum_{\lambda=1}^{2} \boldsymbol{\varepsilon}_{\mathbf{k}, \lambda} \otimes \boldsymbol{\varepsilon}_{\mathbf{k}, \lambda}^{*}\left(W\left[A_{k, \lambda}\left(\eta_{i}\right), A_{k, \lambda}^{*}\left(\eta_{i}\right)\right]-\frac{2 i k}{\varepsilon\left(\eta_{i}\right)}\right)=0 .
$$

We now take $\eta_{i}$ as the initial time, namely when inflation begins, and we assume that $A_{k, 1}\left(\eta_{i}\right)=A_{k, 2}\left(\eta_{i}\right)$. This choice implies that $W\left[A_{k, \lambda}\left(\eta_{i}\right), A_{k, \lambda}^{*}\left(\eta_{i}\right)\right]$ does not depend on $\lambda$. Consequently, Eq. (53) is satisfied only if

$W\left[A_{k, \lambda}\left(\eta_{i}\right), A_{k, \lambda}^{*}\left(\eta_{i}\right)\right]=\frac{2 i k}{\varepsilon\left(\eta_{i}\right)}$,

in which case we have

$W\left[A_{k, \lambda}(\eta), A_{k, \lambda}^{*}(\eta)\right]=\frac{2 i k}{\varepsilon(\eta)}$,

for all $\eta$. Equation (55) represents the desired condition that must be satisfied by any solution $A_{k, \lambda}(\eta)$ of the equation of motion in order to have a consistent quantization of the electromagnetic field.

\subsection{Bunch-Davies normalized solutions}

Let us assume, for the sake of simplicity, that the external tensor $\left(k_{F}\right)_{\nu}^{\mu}$ is constant during inflation ${ }^{3}$ (so that $\rho_{k}$ and $p_{K}$ are constant as well), and that the coupling function $f(\phi)$ evolves in time following a simple power law,

$f(\phi(\eta))=f_{i}\left(\frac{\eta}{\eta_{i}}\right)^{\gamma}$

where $f_{i}=f\left(\phi\left(\eta_{i}\right)\right)$, and $\gamma$ is a free index. In this case, the permittivity $\varepsilon$ and the permeability $\mu$ evolve in time as $\eta^{\gamma}$, while the refractive index $n$ is a constant. The solution of Eq. (46) is then easily found,

$A_{k, \lambda}=(-k \eta)^{v}\left[c_{k}^{(1)} H_{v}^{(1)}(-k \eta / n)+c_{k}^{(2)} H_{v}^{(2)}(-k \eta / n)\right]$,

where $v=(1-\gamma) / 2, H_{v}^{(1,2)}(x)$ are the Hankel functions of first and second kind, respectively, and $c_{k}^{(1,2)}$ are integration constants. The latter can be fixed by the choice of the vacuum, which we take to be the Bunch-Davies vacuum [13,14]. It reduces to the standard Minkowski vacuum in the short

\footnotetext{
${ }^{3}$ It is important to stress that $\left(k_{F}\right)_{\mu \nu}$ must evolve after inflation in such a way to be consistent with current experimental limits on Lorentzviolation coefficients [115].
} 
wavelength limit, $k \rightarrow \infty$. To find it, let us re-scale the electromagnetic field as

$\psi_{k, \lambda}=\frac{A_{k, \lambda}}{\sqrt{\left|W\left[A_{k, \lambda}, A_{k, \lambda}^{*}\right]\right|}}$.

Inserting Eq. (58) in Eq. (46), we see that the re-scaled $\psi$ modes satisfy the equation of motion

$\ddot{\psi}_{k, \lambda}=U_{k} \psi_{k, \lambda}$,

where we have defined

$U_{k}=-\frac{k^{2}}{n^{2}}+\frac{1}{\sqrt{\varepsilon}} \frac{\partial^{2}}{\partial \eta^{2}} \sqrt{\varepsilon}$.

Let us observe that Eq. (59) is formally equal to the zeromode, one-dimensional Schrodinger equation with potential energy $U_{k}, \eta$ taking the place of the spatial coordinate, and $k$ playing the role of a free constant parameter. If $\psi_{k, \lambda}^{(1)}$ and $\psi_{k, \lambda}^{(2)}$ are any two solutions of Eq. (59), the following inner product is conserved:

$\left\langle\psi_{k, \lambda}^{(1)} \mid \psi_{k, \lambda}^{(2)}\right\rangle=-i\left(\psi_{k, \lambda}^{(1)} \dot{\psi}_{k, \lambda}^{(2)}-\dot{\psi}_{k, \lambda}^{(1)} \psi_{k, \lambda}^{(2)}\right)$.

Moreover, using Eq. (55), we see that $\psi$-modes are normalized as

$\left\langle\psi_{k, \lambda} \mid \psi_{k, \lambda}^{*}\right\rangle=1$.

For $k \rightarrow \infty$, the potential energy is dominated by the first term in the right-hand-side of Eq. (60). Therefore, the positive-frequency solution of Eq. (59) in the short wavelength limit is $\psi_{k, \lambda}=c_{k} \mathrm{e}^{-i \omega_{k} \eta}$, where $\omega_{k}=k / n$ and $c_{k}$ is an integration constant. The latter is fixed the normalization condition (62), $c_{k}=1 / \sqrt{2 \omega_{k}}$, so that $\psi_{k, \lambda}=1 / \sqrt{2 \omega_{k}} \mathrm{e}^{-i \omega_{k} \eta}$. Accordingly, the Minkowski vacuum $(k \rightarrow \infty)$ is defined by the normalized electromagnetic field solution

$A_{k, \lambda}=\sqrt{Z} \mathrm{e}^{-i \omega_{k} \eta}$,

where $Z=\sqrt{\mu / \varepsilon}$ is, in the language of the electrodynamics of continuous media, the wave impedance of the medium.

Equation (57) must then reduce to Eq. (63) in the limit $k \rightarrow \infty$. This happens only if $c_{k}^{(1)}=\left[\pi \mathrm{e}^{i \pi(v+1 / 2)}\left(-k \eta_{i}\right)^{\gamma} /\right.$ $\left.2 \varepsilon\left(\eta_{i}\right)\right]^{1 / 2}$ and $c_{k}^{(2)}=0$, in which case

$A_{k, \lambda}=\sqrt{\frac{\pi}{2}} \mathrm{e}^{i \frac{\pi}{2}(v+1 / 2)} \sqrt{Z} \sqrt{-\omega_{k} \eta} H_{v}^{(1)}\left(-\omega_{k} \eta\right)$

is the desired Bunch-Davies vacuum normalized solution.

\section{Backreaction on inflation}

We now draw our attention to the electromagnetic backreaction on inflation in the model described by Lagrangian (25). We will find the conditions under which such a backreaction is completely negligible.
Let us first observe that Lagrangian density (34) can be conveniently rewritten as

$\mathcal{L}_{\mathrm{em}}=-\frac{1}{4} F_{\mu \nu} \mathcal{D}^{\mu \nu}$,

with $\mathcal{D}_{\mu \nu}$ given by Eq. (38). The electromagnetic energymomentum tensor is obtained by inserting Eq. (65) in Eq. (7). We find

$\left(T_{\mathrm{em}}\right)_{\mu \nu}=\left(T_{\mathrm{med}}\right)_{\mu \nu}+\left(T_{\mathcal{X}}\right)_{\mu \nu}$.

Here,

$\left(T_{\text {med }}\right)_{\mu \nu}=F_{\alpha\{\mu} \mathcal{D}_{\nu\}}^{\alpha}+\frac{1}{4} F_{\alpha \beta} \mathcal{D}^{\alpha \beta} g_{\mu \nu}$

is the standard electromagnetic energy-momentum tensor in a medium described by the displacement tensor (38) [curly brackets $\{\ldots\}$ indicate a symmetrization of the indices enclosed, e.g., $T_{\mu_{1} \ldots\left\{\mu_{i} \mu_{j}\right\} \ldots \mu_{n}}=\frac{1}{2}\left(T_{\mu_{1} \ldots \mu_{i} \mu_{j} \ldots \mu_{n}}+\right.$ $\left.\left.T_{\mu_{1} \ldots \mu_{j} \mu_{i} \ldots \mu_{n}}\right)\right]$, and

$\left(T_{\mathcal{X}}\right)_{\mu \nu}=\frac{1}{4} \mathcal{X}_{\gamma \delta \mu \nu}^{\alpha \beta} F_{\alpha \beta} F^{\gamma \delta}$.

The rank-five tensor

$\mathcal{X}_{\gamma \delta \mu \nu}^{\alpha \beta}=\frac{2}{\sqrt{-g}} \frac{\delta}{\delta g^{\mu \nu}} \int \mathrm{d}^{4} x \sqrt{-g} \chi_{\gamma \delta}^{\alpha \beta}$

is antisymmetric on the first two and second two indices, symmetric in the last two indices, and it is symmetric for the interchange of the first and second pair of indices.

When the susceptibility tensor has the form of Eq. (35), the electromagnetic energy-momentum tensor can be written, in its full form, as

$$
\begin{aligned}
\left(T_{\mathrm{em}}\right)_{\mu \nu}= & f(\phi)\left[F_{\alpha \mu} F_{\nu}^{\alpha}+\frac{1}{4} F_{\alpha \beta} F^{\alpha \beta} g_{\mu \nu}\right. \\
& -\frac{1}{2}\left(\xi_{1}+\xi_{2}\right)\left(k_{F}\right)_{\mu \nu} F_{\alpha \beta} F^{\alpha \beta} \\
& -2 \xi_{2}\left(k_{F}\right)_{\beta}^{\alpha} F_{\alpha \mu} F_{\nu}{ }^{\beta}+4 \xi_{2}\left(k_{F}\right)_{\{\mu}^{\alpha} F_{\nu\} \beta} F_{\alpha}{ }^{\beta} \\
& \left.-\xi_{2}\left(k_{F}\right)_{\beta}^{\alpha} F_{\alpha \gamma} F^{\beta \gamma} g_{\mu \nu}\right] .
\end{aligned}
$$

Due to symmetry, we find that the only (possible) nonnull components of the vacuum expectation value of the electromagnetic energy-momentum tensor, $\left\langle\left(T_{\mathrm{em}}\right)_{\nu}^{\mu}\right\rangle$, are the electromagnetic energy density, $\left\langle\left(T_{\mathrm{em}}\right)_{0}^{0}\right\rangle$, and $\left\langle\left(T_{\mathrm{em}}\right)_{j}^{i}\right\rangle=$ $\frac{1}{3}\left(\left\langle\left(T_{\mathrm{em}}\right)_{\mu}^{\mu}\right\rangle-\left\langle\left(T_{\mathrm{em}}\right)_{0}^{0}\right\rangle\right) \delta_{i j}$. Here, $\left(T_{\mathrm{em}}\right)_{\mu}^{\mu}$ is the trace of the electromagnetic energy-momentum tensor, which is, in general, different from zero due to the coupling of the photon to the background tensor $\left(k_{F}\right)_{v}^{\mu}$. In particular, we have

$$
\begin{aligned}
\rho_{\mathrm{em}} & =\frac{\varepsilon}{2}\left\langle\mathbf{E}^{2}\right\rangle+\frac{1}{2 \mu}\left\langle\mathbf{B}^{2}\right\rangle+\left\langle\left(T_{\mathcal{X}}\right)_{0}^{0}\right\rangle, \\
T_{\mathrm{em}} & =\left\langle\left(T_{\mathcal{X}}\right)_{\mu}^{\mu}\right\rangle,
\end{aligned}
$$


where we have defined

$\rho_{\mathrm{em}}=\left\langle\left(T_{\mathrm{em}}\right)_{0}^{0}\right\rangle, \quad T_{\mathrm{em}}=\left\langle\left(T_{\mathrm{em}}\right)_{\mu}^{\mu}\right\rangle$.

When the susceptibility tensor has the form of Eq. (35), we have

$$
\begin{aligned}
& \left\langle\left(T_{\mathcal{X}}\right)_{0}^{0}\right\rangle=f(\phi) \rho_{K}\left[-\left(\xi_{1}-\xi_{2}\right)\left\langle\mathbf{E}^{2}\right\rangle+\left(\xi_{1}+\xi_{2}\right)\left\langle\mathbf{B}^{2}\right\rangle\right], \\
& \left\langle\left(T_{\mathcal{X}}\right)_{i}^{i}\right\rangle=f(\phi) p_{K}\left[\left(3 \xi_{1}+\xi_{2}\right)\left\langle\mathbf{E}^{2}\right\rangle-\left(3 \xi_{1}-\xi_{2}\right)\left\langle\mathbf{B}^{2}\right\rangle\right] .
\end{aligned}
$$

The vacuum expectation value of the squared magnetic and electric fields operator are easily found:

$$
\begin{aligned}
& \left\langle\mathbf{B}^{2}\right\rangle=\int_{0}^{\infty} \frac{\mathrm{d} k}{k} \mathcal{P}_{\mathrm{B}}(k, \eta), \\
& \left\langle\mathbf{E}^{2}\right\rangle=\int_{0}^{\infty} \frac{\mathrm{d} k}{k} \mathcal{P}_{\mathrm{E}}(k, \eta),
\end{aligned}
$$

where $\mathcal{P}_{\mathrm{B}}(k, \eta)$ and $\mathcal{P}_{\mathrm{E}}(k, \eta)$ are the so-called magnetic and electric power spectra,

$\mathcal{P}_{\mathrm{B}}(k, \eta)=\sum_{\lambda=1}^{2} \frac{k^{4}}{4 \pi^{2} a^{4}}\left|A_{k, \lambda}(\eta)\right|^{2}$,
$\mathcal{P}_{\mathrm{E}}(k, \eta)=\sum_{\lambda=1}^{2} \frac{k^{2}}{4 \pi^{2} a^{4}}\left|\dot{A}_{k, \lambda}(\eta)\right|^{2}$.

Defining also the electromagnetic energy density spectrum, $\rho_{\mathrm{em}}(k, \eta)$, and the electromagnetic trace spectrum, $T_{\mathrm{em}}(k, \eta)$, through

$$
\begin{aligned}
& \rho_{\mathrm{em}}(\eta)=\int_{0}^{\infty} \frac{\mathrm{d} k}{k} \rho_{\mathrm{em}}(k, \eta), \\
& T_{\mathrm{em}}(\eta)=\int_{0}^{\infty} \frac{\mathrm{d} k}{k} T_{\mathrm{em}}(k, \eta),
\end{aligned}
$$

we recast Eqs. (71) and (72) as

$$
\begin{aligned}
& \rho_{\mathrm{em}}(k, \eta)=\frac{1}{2} \tau_{1} \mathcal{P}_{\mathrm{E}}+\frac{1}{2} \tau_{2} \mathcal{P}_{\mathrm{B}}, \\
& T_{\mathrm{em}}(k, \eta)=\tau_{3} \mathcal{P}_{\mathrm{E}}+\tau_{4} \mathcal{P}_{\mathrm{B}},
\end{aligned}
$$

where we have defined

$$
\begin{aligned}
\tau_{1} & =\varepsilon-2\left(\xi_{1}-\xi_{2}\right) f(\phi) \rho_{K}, \\
\tau_{2} & =\mu^{-1}+2\left(\xi_{1}+\xi_{2}\right) f(\phi) \rho_{K}, \\
\tau_{3} & =\varepsilon-f(\phi), \\
\tau_{4} & =f(\phi)-\mu^{-1} .
\end{aligned}
$$

Let us now specialize our results to the case of de Sitter spacetime and for large-scale, superhorizon modes. Inserting the asymptotic expansion for $-k \eta \rightarrow 0$ of the solution (64) in Eqs. (78) and (79), we get

$$
\begin{aligned}
& \mathcal{P}_{\mathrm{B}}(k, \eta)=\frac{\left|c_{\nu}\right|^{2}}{4 \pi} Z n^{4}\left(-\omega_{k} \eta\right)^{5+2 v} H^{4}, \\
& \mathcal{P}_{\mathrm{E}}(k, \eta)=\frac{4 v^{2}}{(-k \eta)^{2}} \mathcal{P}_{\mathrm{B}}(k, \eta),
\end{aligned}
$$

respectively. We are principally interested in the case of a scaling-invariant magnetic spectrum (the general case goes along the same lines as below), so that we take $v=-5 / 2$ [corresponding to $\gamma=6$ in Eq. (56)]. In this case, we have

$\mathcal{P}_{\mathrm{B}}(k, \eta)=\frac{9}{2 \pi^{2}} Z n^{4} H^{4}$,

$\mathcal{P}_{\mathrm{E}}(k, \eta)=\frac{225}{2 \pi^{2}} Z n^{4} \frac{H^{4}}{(-k \eta)^{2}}$.

Looking at Eqs. (82), (83), (90), and (91), and observing that $Z=n / \varepsilon \sim 1 / f(\phi)$, we conclude, following the discussion in Sect. 3.2, that the electromagnetic back-reaction on inflation is not generally negligible. This conclusion could be avoided if the coefficients $\tau_{1}$ and $\tau_{3}$, which enter in the definition of the electric part of the electromagnetic energymomentum tensor, are vanishing. This happens only if the background tensor $\left(k_{F}\right)_{v}^{\mu}$ assumes a particular form, which we are now going to determine. Assuming that $\tau_{3}=0$, we straightforwardly get

$\varepsilon=f(\phi), \quad \mu=\frac{n^{2}}{f(\phi)}, \quad Z=\frac{n}{f(\phi)}$.

The above equations, when combined with the condition $\tau_{1}=0$ and Eq. (31), give

$\rho_{K}=\frac{1}{2\left(\xi_{1}-\xi_{2}\right)}, \quad p_{K}=\frac{1}{2\left(3 \xi_{1}+\xi_{2}\right)}$,

which determine the form of $\left(k_{F}\right)_{v}^{\mu}$ in Eq. (27) as a function of $\xi_{i}$ and, accordingly,

$n=\sqrt{\frac{\left(\xi_{1}-\xi_{2}\right)\left(3 \xi_{1}+\xi_{2}\right)}{3\left(\xi_{1}-\xi_{2}\right)^{2}-4 \xi_{2}^{2}}}$.

Imposing the reality condition, $n^{2}>0$, we find

$\xi_{1}=0 \quad$ or $\quad \xi_{2}=0 \quad$ or $\quad \frac{\xi_{1}}{\xi_{2}} \in \mathbb{X}$

where

$$
\begin{aligned}
\mathbb{X}= & \left(-\infty,-\frac{1}{3}\right) \cup\left(1-\frac{2}{\sqrt{3}}, 0\right) \cup(0,1) \\
& \cup\left(1+\frac{2}{\sqrt{3}},+\infty\right) .
\end{aligned}
$$


Taking into account Eqs. (92) and (93), we find

$\mathcal{P}_{\mathrm{B}}(k, \eta)=\frac{9 n^{5}}{2 \pi^{2} f(\phi)} H^{4}$,

$\rho_{\mathrm{em}}(k, \eta)=\frac{9 n^{5} \Upsilon_{1}}{2 \pi^{2}} H^{4}$,

$T_{\mathrm{em}}(k, \eta)=\frac{9 n^{5} \Upsilon_{2}}{2 \pi^{2}} H^{4}$,

where we have defined

$\Upsilon_{1}=\frac{\xi_{1}\left(3 \xi_{1}-\xi_{2}\right)}{\left(\xi_{1}-\xi_{2}\right)\left(3 \xi_{1}+\xi_{2}\right)}$,

$\Upsilon_{2}=\frac{4 \xi_{1} \xi_{2}}{\left(\xi_{1}-\xi_{2}\right)\left(3 \xi_{1}+\xi_{2}\right)}$.

We observe that all the three of the above spectra are scaling-invariant, and that $\rho_{\mathrm{em}}(k, \eta)$ and $T_{\mathrm{em}}(k, \eta)$ are timeindependent, while the time dependence of $\mathcal{P}_{B}(k, \eta)$ is all encoded in $f(\phi)$.

Finally, imposing $\left\langle\left(T_{\mathrm{em}}\right)_{v}^{\mu}\right\rangle \ll\left(T_{\mathrm{inf}}\right)_{v}^{\mu}$, we get

$$
\begin{aligned}
& \frac{\left|\rho_{\mathrm{em}}\right|}{\rho_{\text {inf }}}=32 n^{5}\left|\Upsilon_{1}\right|\left(\frac{M}{m_{\mathrm{Pl}}}\right)^{4} \ll 1, \\
& \frac{\left|T_{\mathrm{em}}\right|}{\rho_{\text {inf }}}=32 n^{5}\left|\Upsilon_{2}\right|\left(\frac{M}{m_{\mathrm{Pl}}}\right)^{4} \ll 1,
\end{aligned}
$$

which are the wanted conditions that must be satisfied in order to have a negligible electromagnetic back-reaction on inflationary dynamics.

\section{Actual magnetic field}

We have seen that inflation is able to produce superhorizon magnetic field fluctuations whose intensity is given by the magnetic power spectrum (97). For the following discussion, it is useful to define the magnetic field strength on the scale $\lambda=1 / k$ as

$B(\lambda, \eta)=\sqrt{\mathcal{P}_{\mathrm{B}}(1 / \lambda, \eta)}$.

At the end of inflation, we have then the scale-invariant magnetic field

$B_{\text {end }}=\frac{3}{\sqrt{2} \pi}\left(\frac{n^{5}}{f_{\text {end }}}\right)^{1 / 2} H^{2}$,

where $B_{\text {end }}=B\left(\lambda, \eta_{\text {end }}\right)$ and $f_{\text {end }}=f\left(\phi\left(\eta_{\text {end }}\right)\right)$.

Such a field will evolve from the end of inflation until today. In this section, we will find the actual value of the inflation-produced magnetic field as a function of the free parameters of the model, namely the constants $\xi_{1}$ and $\xi_{2}$ [the other two free parameters, $\rho_{K}$ and $p_{K}$, are assumed to be fixed by Eq. (93)] and, consequently, find the regions in the parameter space $\left(\xi_{1}, \xi_{2}\right)$ where it satisfies both the constraint in Eq. (2) and those in Eqs. (102) and (103).

\subsection{Evolution after reheating}

In order to find the present intensity of the magnetic field, we must evolve it from the end of inflation until the present time $\eta_{0}$. As in Sect. 3.2, and to simplify the analysis, we consider the case of instantaneous reheating. After the end of reheating (which corresponds in this case to the end of inflation and the beginning of radiation era), the dynamics of the inflation-produced electromagnetic field is governed by standard Lagrangian

$\mathcal{L}_{\mathrm{em}}=\mathcal{L}_{\mathrm{M}}+j^{\mu} A_{\mu}$,

since $f(\phi(\eta))=1$ after inflation. Here, we have assumed, for the sake of simplicity, that the background tensor field is vanishingly small for $\eta>\eta_{\text {end }}$. This assures that the experimental constraints on the coefficients of the Lorentz violation $\left(k_{F}\right)_{\mu \nu \alpha \beta}$ are automatically fulfilled [115].

The post-inflationary external electric current $j^{\mu}$ is vanishing on superhorizon scales due to causality $[61,62]$, while inside the horizon it can be written as $j_{\mu}=\left(0,-\tilde{\sigma}_{\mathrm{c}} \mathbf{E}\right)$, where $\tilde{\sigma}_{\mathrm{c}}=a \sigma_{\mathrm{c}}$ is the comoving conductivity and $\sigma_{\mathrm{c}}$ is the standard conductivity of the plasma. Accordingly, the equation of the motion for the comoving magnetic field $a^{2} \mathbf{B}$, also known as the magnetic flux $\mathbf{F}$, is

$\ddot{\mathbf{F}}-\nabla^{2} \mathbf{F}=0$

for modes that live outside the horizon, and [15]

$\ddot{\mathbf{F}}-\nabla^{2} \mathbf{F}=-\tilde{\sigma}_{\mathrm{c}} \dot{\mathbf{F}}$

for subhorizon modes. Going to Fourier space, $\mathbf{F}_{\mathbf{k}}(\eta)=$ $\int \mathrm{d}^{3} x \mathrm{e}^{i \mathbf{k x}} \mathbf{F}(\mathbf{x}, \eta)$, and observing that $\left|k^{2} \mathbf{F}_{\mathbf{k}}\right| / \ddot{\mathbf{F}}_{\mathbf{k}} \mid \sim(-k \eta)^{2}$, we find that superhorizon magnetic modes $(-k \eta \ll 1)$ evolve according to $\ddot{\mathbf{F}}_{\mathbf{k}}=0$, so that they scale adiabatically, $\mathbf{B} \propto a^{-2}$. Modes inside the horizon $(-k \eta \gg 1)$, instead, evolve according to the so-called (comoving) autoinduction equation (see, e.g., [116]), $\dot{\mathbf{F}}_{\mathbf{k}}=-\left(k^{2} / \tilde{\sigma}_{\mathrm{c}}\right) \mathbf{F}_{\mathbf{k}}$. The solution of the above equation is

$\mathbf{F}_{\mathbf{k}}(\eta)=\mathbf{F}_{\mathbf{k}}\left(\eta_{\mathrm{RH}}\right) \mathrm{e}^{-k^{2} \ell_{\mathrm{d}}^{2} /(2 \pi)^{2}}$,

where

$\ell_{\mathrm{d}}(\eta)=2 \pi \sqrt{\int_{\eta_{\mathrm{RH}}}^{\eta} \frac{\mathrm{d} \eta^{\prime}}{\tilde{\sigma}_{\mathrm{c}}\left(\eta^{\prime}\right)}}$

is the comoving dissipation length and $\mathrm{RH}$ indicates the time of reheating. Accordingly, modes with wave number $k \ll$ $2 \pi / \ell_{\mathrm{d}}$ evolve adiabatically, while modes with $k \gg 2 \pi / \ell_{\mathrm{d}}$ are dissipated. ${ }^{4}$

\footnotetext{
${ }^{4}$ Here, we are neglecting possible effects of magnetohydrodynamic turbulence that could take place in correspondence of the electroweak and/or quark-hadron (QCD) phase transitions, and that could affect the evolution of the inflation-generated magnetic field [116-134]. However, it has been recently shown [135] that a scaling-invariant magnetic field
} 
Putting all together, we conclude that, during the expansion of the Universe after reheating, magnetic modes are washed out on scales below the dissipation length and diluted adiabatically on larger scales. However, the actual dissipation length is very small compared to the scale of interest for cosmic magnetic fields.

To see this, we firstly remember the conductivity $\sigma_{\mathrm{c}}$ depends, generally, on the temperature $T$ [15]. In the radiation-dominated era, and for temperatures much greater than the electron mass $m_{e}$, we have $\sigma_{\mathrm{c}}(T) \sim T / e^{2}$ [15], where $e$ is the absolute value of the electric charge. After the epoch of $e^{+} e^{-}$annihilation $\left(T_{\mathrm{anh}} \sim m_{e}\right)$, the conductivity is given by $\sigma_{\mathrm{c}}(T) \sim\left(T / e^{2}\right) \sqrt{T / m_{e}}$ [136], while in the matter-dominated era $\left(T<T_{\text {eq }} \simeq 3 \mathrm{eV}\right)$, and after electrons and ions have recombined $\left(T_{\text {rec }} \simeq 0.3 \mathrm{eV}\right)$, it drops to the constant value $\sigma_{\mathrm{c}}(T) \sim 10^{-13} m_{e} / e^{2} \simeq 8 \times 10^{8} \mathrm{~s}^{-1}$ [15].

Taking into account that $\eta \propto a$ and $\eta \propto a^{1 / 2}$ in the radiation-dominated and matter-dominated eras, respectively, and that $a \propto g_{* S}^{-1 / 3} T^{-1}$ after reheating, where $g_{* S}(T)$ is the effective number of entropy degrees of freedom at the temperature $T$ [137], we conveniently split the integral in Eq. (111), evaluated at the present time, in four integrals, $\int_{\eta_{\mathrm{RH}}}^{\eta_{0}} \mathrm{~d} \eta / \tilde{\sigma}_{\mathrm{c}}=I_{1}+I_{2}+I_{3}+I_{4}$. Here, $I_{1}=\int_{\eta_{\mathrm{RH}}}^{\eta_{\mathrm{anh}}} \mathrm{d} \eta / \tilde{\sigma}_{\mathrm{c}}$, $I_{2}=\int_{\eta_{\mathrm{anh}}}^{\eta_{\mathrm{eq}}} \mathrm{d} \eta / \tilde{\sigma}_{\mathrm{c}}, I_{3}=\int_{\eta_{\mathrm{eq}}}^{\eta_{\mathrm{rec}}} \mathrm{d} \eta / \tilde{\sigma}_{\mathrm{c}}$, and $I_{4}=\int_{\eta_{\mathrm{rec}}}^{\eta_{0}} \mathrm{~d} \eta / \tilde{\sigma}_{\mathrm{c}}$. Since $I_{1} / I_{2} \sim\left(T_{\text {eq }} / m_{e}\right)^{3 / 2} \sim 10^{-8}, I_{2} / I_{3} \sim T_{\text {rec }} / T_{\text {eq }} \sim$ $10^{-1}$, and $I_{3} / I_{4} \sim 10^{-13}\left(m_{e} / T_{\text {rec }}\right)^{3 / 2} \sim 10^{-4}$, the integral $I_{4}$ dominates over the other three in the expression for the actual dissipation length. Accordingly, we have

$\ell_{\mathrm{d}}\left(t_{0}\right) \simeq 2 \pi\left(\frac{3 t_{0}}{\sigma_{c}}\right)^{1 / 2}\left(\frac{t_{0}}{t_{\mathrm{rec}}}\right)^{1 / 6} \simeq 10^{-2} \mathrm{pc}$,

where we used the fact that in the matter-dominated era $a(t) \simeq\left(t / t_{0}\right)^{2 / 3}$ and then $\eta(t) \simeq 3 t_{0}\left(t / t_{0}\right)^{1 / 3}$, and $t_{\mathrm{rec}} \simeq$ $8 \times 10^{12} \mathrm{~s}[137]$.

\subsection{Actual magnetic field strength}

As anticipated, the actual dissipation length is negligibly small compared to the scale of interest for cosmic magnetic fields, which is of order of $1 \mathrm{Mpc}$. We conclude that the inflation-produced magnetic field evolve adiabatically, from the time of reheating until today. Its actual intensity is then

$B_{0}=B_{\text {end }}\left(\frac{g_{* S, 0}}{g_{* S, \mathrm{RH}}}\right)^{2 / 3}\left(\frac{T_{0}}{T_{\mathrm{RH}}}\right)^{2} \cos \theta_{\mathrm{W}}$,

where $B_{0}=B\left(\lambda, \eta_{0}\right), g_{* S, 0}=g_{* S}\left(T_{0}\right)=43 / 11$ [138], $g_{* S, \mathrm{RH}}=g_{* S}\left(T_{\mathrm{RH}}\right)[138], T_{0} \simeq 2.37 \times 10^{-4} \mathrm{eV}$ [137] is the actual temperature, and $T_{\mathrm{RH}}$ is the reheat temperature.

Footnote 4 continued

stays almost unchanged on scales of cosmological interest, although on smaller scales its spectrum is progressively suppressed.
Above the electroweak phase transition (when we assume inflation is taking place) the $U(1)$ gauge field which is quantum mechanically excited is indeed the hypercharge field, not the electromagnetic one [16]. Below the electroweak phase transition, however, the hypercharge field is projected onto the electromagnetic field, and this gives the cosine of the Weinberg angle $\theta_{\mathrm{W}}$.

The reheat temperature can be related to the energy scale of inflation by observing that the energy density of radiation at the beginning of radiation era, $\rho_{\mathrm{rad}}=\left(\pi^{2} / 30\right) g_{*, \mathrm{RH}} T_{\mathrm{RH}}^{4}$, where $g_{*, \mathrm{RH}}$ is the effective number of degrees of freedom at the time of reheating and can be taken equal to $g_{* S, \mathrm{RH}}[137]$, must be equal to the energy density at the end of inflation. We get $T_{\mathrm{RH}}=\left[30 /\left(\pi^{2} g_{*, \mathrm{RH}}\right)\right]^{1 / 4} M$. Taking $g_{* S, \mathrm{RH}}=427 / 4$ [137], referring to the massless degrees of freedom of the standard model of particle physics above the electroweak scale, the actual, scale-invariant magnetic field is

$B_{0} \simeq 2 \times 10^{-12}\left(\frac{n^{5}}{f_{\text {end }}}\right)^{1 / 2}\left(\frac{M}{10^{16} \mathrm{GeV}}\right)^{2} \mathrm{G}$

Let us now take $f_{\text {end }} \sim 1$ and $M \sim 10^{16} \mathrm{GeV}$. Accordingly, we have

$B_{0} \sim n^{5 / 2} 10^{-12} \mathrm{G}$.

The condition that the electromagnetic back-reaction on inflation is negligible, expressed by Eqs. (102) and (103), becomes

$n^{5}\left|\Upsilon_{1}\right| \ll 10^{11}$,

$n^{5}\left|\Upsilon_{2}\right| \ll 10^{11}$.

Let us now analyze, separately, the three cases in Eq. (95), namely $\xi_{1}=0, \xi_{2}=0$, and $\xi_{1} / \xi_{2} \in \mathbb{X}$.

(i) $\xi_{1}=0$. This corresponds, looking at Lagrangian density (23), to the case where the electromagnetic field is coupled only to the traceless part of the background tensor $\left(k_{F}\right)_{\nu}^{\mu}$. From Eqs. (93), (94), (98), and (99), we get $\rho_{K}=-1 / 2 \xi_{2}$, $p_{K}=-\rho_{K}, n=1, \Upsilon_{1}=0$, and $\Upsilon_{2}=0$, respectively. This gives, in turns, $\left\langle\left(T_{\mathrm{em}}\right)_{v}^{\mu}\right\rangle=0$, so that the electromagnetic back-reaction on inflation is absent. The actual, scalinginvariant magnetic field is of order of $B_{0} \sim 10^{-12} \mathrm{G}$ and it can directly account for the presently observed cosmic magnetic fields.

(ii) $\xi_{2}=0$. This case corresponds to an electromagnetic field coupled only to the scalar part (trace) of $\left(k_{F}\right)_{\nu}^{\mu}$. We have $\rho_{K}=1 / 2 \xi_{1}, p_{K}=\rho_{K} / 3, n=1, \Upsilon_{1}=1$, and $\Upsilon_{2}=0$ (which gives $T_{\mathrm{em}}=0$ ). The electromagnetic backreaction on inflation is, then, completely negligible and, also in this case, the inflation-produced magnetic field can directly explain cosmic magnetization.

(iii) $\xi_{1} / \xi_{2} \in \mathbb{X}$. In Fig. 1, we plot the function $n^{5 / 2}$, entering in the expression of $B_{0}$ in Eq. (114), at the vary- 

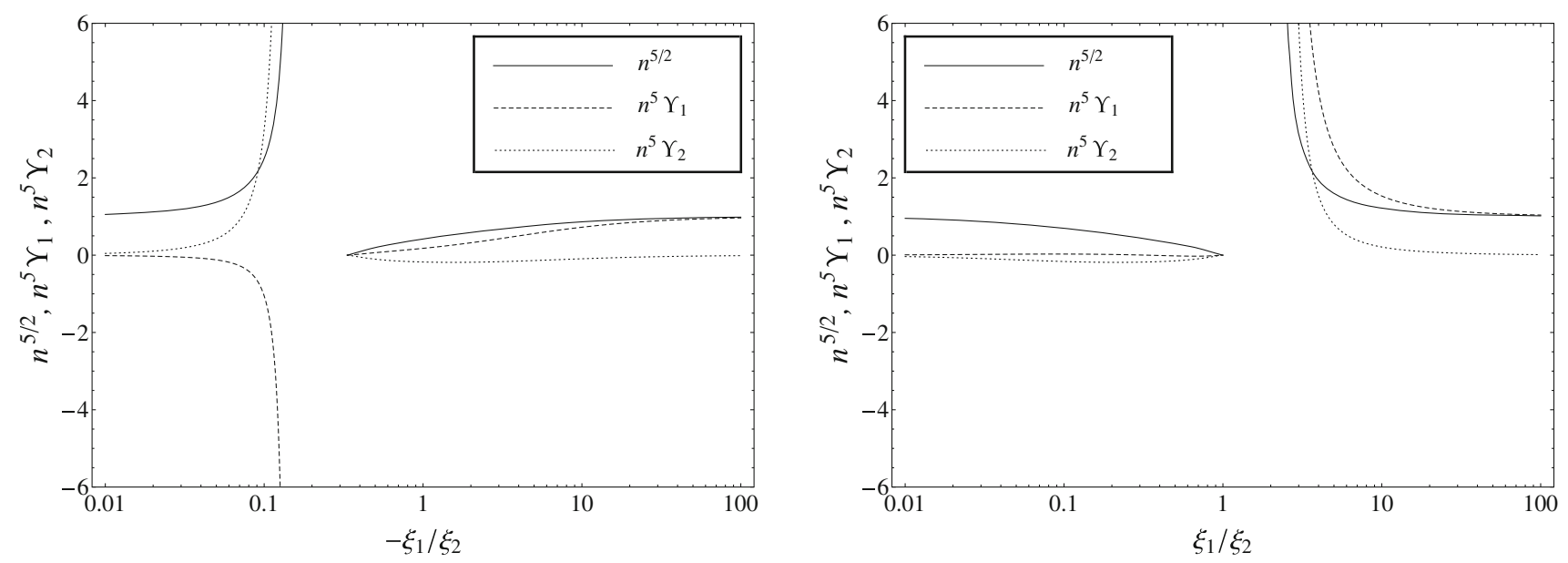

Fig. 1 The quantities $n^{5 / 2}$ (continuous lines), $n^{5} \Upsilon_{1}$ (dashed lines), and $n^{5} \Upsilon_{2}$ (dotted lines) [which appear in Eqs. (114), (115), and (116), respectively], as a function of $\xi_{1} / \xi_{2}$

ing of $\xi_{1} / \xi_{2}$. Remembering the discussion in Sect. 2 [see, in particular, Eq. (2)], we find that, in order to explain cosmic magnetization, the quantity $n^{5 / 2}$ should be in the range $\left[0.1\right.$, few]. This is realized for all values of $\xi_{1} / \xi_{2} \in \mathbb{X}$, with the exclusion of those values very close to the boundary $\partial \mathbb{X}=\{-1 / 3\} \cup\{1-2 / \sqrt{3}\} \cup\{1\} \cup\{1+2 / \sqrt{3}\}$. In fact, $n$ diverges for $\xi_{1} / \xi_{2} \rightarrow-1 / 3$ and $\xi_{1} / \xi_{2} \rightarrow 1+2 / \sqrt{3}$, while it goes to zero for $\xi_{1} / \xi_{2} \rightarrow 1-2 / \sqrt{3}$ and $\xi_{1} / \xi_{2} \rightarrow 1$. When $\xi_{1} / \xi_{2}$ is not so close to the above boundary values, the conditions (115) and (116), which assure that the electromagnetic field does not back-react on the inflationary dynamics, are fulfilled. This is clear from Fig. 1, where we show $n^{5} \Upsilon_{1}$ and $n^{5} \Upsilon_{2}$ with varying $\xi_{1} / \xi_{2}$. We conclude that, apart from some particular values of $\xi_{1} / \xi_{2}$, the inflation-produced magnetic field can be, also in this case, at the origin of cosmic magnetic fields.

\section{Additional conditions on $\left(T_{\mathrm{em}}\right)_{v}^{\mu}$}

Let us now impose some physically "reasonable" conditions on the inflation-produced electromagnetic energymomentum tensor. Some of these conditions, as the positivity of the energy, are often assumed to be "necessary" in the literature. It is worth noticing, however, that there exist examples of physically "reasonable" matter that violate some or of all of them. For example, all the conditions that we are going to discuss are violated in particular setups of the Casimir effect [13], and even the inflaton violates the strong energy condition (discussed below) when it drives de Sitter inflation.

Weak energy condition. Looking at the left panel of Fig. 1, we see that the quantity $n^{5} \Upsilon_{1}$ is negative for $\xi_{1} / \xi_{2}<-1 / 3$. This corresponds to have a negative electromagnetic energy density on large superhorizon scales during inflation [see Eq. (98)]. On these scales, we expect that the electromag- netic field behaves classically, so that one could wonder if having classical negative energies is reasonable physically. Let us then impose the condition of positivity of the energy. In a general-covariant formulation, this condition is known as "weak energy condition" and it is, indeed, a condition on the energy-momentum tensor. In our specific case, the electromagnetic energy-momentum tensor can be written as

$\left\langle\left(T_{\mathrm{em}}\right)_{v}^{\mu}\right\rangle=\operatorname{diag}\left(\rho_{\mathrm{em}},-p_{\mathrm{em}},-p_{\mathrm{em}},-p_{\mathrm{em}}\right)$.

This is the energy-momentum tensor of a (isotropic) perfect fluid of type I (according to the Hawking-Ellis classification [139]) with energy density $\rho_{\mathrm{em}}$ and pressure density $p_{\mathrm{em}}=-\left\langle\left(T_{\mathrm{em}}\right)_{i}^{i}\right\rangle$ (no sum on $i$ ). For perfect fluids, the weak energy condition states that [139]

$\rho_{\mathrm{em}} \geq 0, \quad \rho_{\mathrm{em}}+p_{\mathrm{em}} \geq 0$.

These supplementary conditions, if applied to Eq. (117), would narrow the domain (96) to $\mathbb{X}=(-\infty,-1 / 3) \cup$ $(0,1 / 3) \cup(1+2 / \sqrt{3},+\infty)$.

Trace condition. It is well known that the trace of the energy-momentum tensor for a system of point-like, electromagnetic interacting particles is non-negative [140]. This condition is sometimes assumed to be valid also for other interacting systems in Nature [140]. If we require that

$T_{\mathrm{em}} \geq 0$,

the domain (96) would reduce to $\mathbb{X}=(1-2 / \sqrt{3}, 0) \cup(1+$ $2 / \sqrt{3},+\infty)$.

There are other restrictions on the energy-momentum tensor conjectured to hold for all physically reasonable matter. Those are the strong and dominant energy conditions. (The physical significance of these conditions is, respectively, and roughly speaking, that matter must gravitate toward matter, and that energy must either be non-negative and not flow faster than light [139].) 

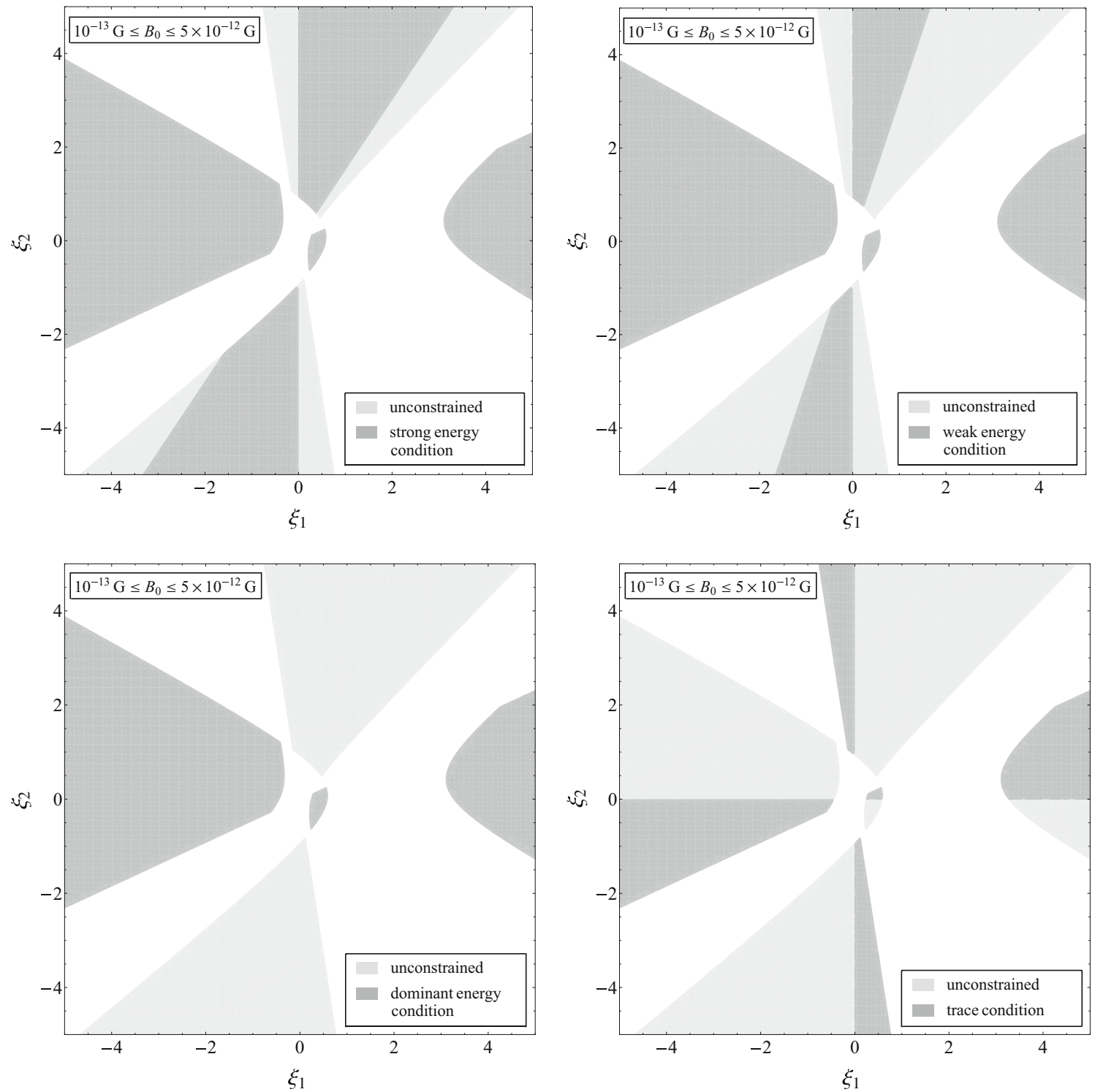

Fig. 2 Regions in the parameter space $\left(\xi_{1}, \xi_{2}\right)$, with $\xi_{1} / \xi_{2} \in \mathbb{X}$ [see Eq. (96)], where $10^{-13} \mathrm{G} \leq B_{0} \leq 5 \times 10^{-12} \mathrm{G}$ and electromagnetic back-reaction on inflation is completely negligible (light gray areas). In the shrunk dark gray regions, a specific supplementary condition

on the electromagnetic energy-momentum has been imposed (from up to down and from left to right: strong energy condition, weak energy condition, dominant energy condition, and trace condition)

Strong energy condition. This condition requires that [139]

$\rho_{\mathrm{em}} \geq 0, \quad \rho_{\mathrm{em}}+3 p_{\mathrm{em}} \geq 0$,

and would reduce the domain (96) to $\mathbb{X}=(-\infty,-1 / 3) \cup$ $(0,2 / 3) \cup(1+2 / \sqrt{3},+\infty)$.

Dominant energy condition. This condition imposes [139]

$\rho_{\mathrm{em}} \geq\left|p_{\mathrm{em}}\right|$

and, if applied, it would shrink the domain (96) to $\mathbb{X}=$ $(-\infty,-1 / 3) \cup(1+2 / \sqrt{3},+\infty)$.

If we impose simultaneously all the above conditions, we would reduce the domain (96) to $\mathbb{X}=(1+2 / \sqrt{3},+\infty)$. This means that the only "surviving" part of Fig. 1 would be the right branch in its right panel.

The light gray areas in Fig. 2 show the regions in the parameter space $\left(\xi_{1}, \xi_{2}\right)$ where $10^{-13} \mathrm{G} \leq B_{0} \leq 5 \times 10^{-12} \mathrm{G}$ and electromagnetic back-reaction on inflation is completely negligible. The dark gray areas represent, instead, the shrunk regions where a specific supplementary condition on the electromagnetic energy-momentum has been imposed (from up 
to down and from left to right: strong energy condition, weak energy condition, dominant energy condition, and trace condition).

\section{Curvature perturbations}

Recently enough, it has been pointed out in the literature that the production of electromagnetic fields during inflation may significantly affect the primordial spectrum of both scalar and tensor curvature perturbations (see, e.g., [80,141-143]). In order to have a self-consistent model of inflationary magnetogenesis, then, we have to check that the curvature perturbations introduced by the inflation-produced electromagnetic field are compatible with $\mathrm{CMB}$ results.

\subsection{Scalar curvature perturbation}

In order to find how a primordial magnetic field can generate curvature perturbations, let us consider the curvature perturbation $\zeta(t, \mathbf{x})$ on the uniform energy density hypersurface [144] on which $\delta \rho(t, \mathbf{x})=0$, where $\delta \rho$ is the energy density perturbation, and $t$ is the cosmic time. The curvature perturbation, as a function of the scale factor $a(t, \mathbf{x})$, is [144]

$\zeta(t, \mathbf{x})=\ln a(t, \mathbf{x})-\ln a(t)$,

where $a(t)$ is the global scale factor, namely the one introduced in the unperturbed metric (8). On super-Hubble scales, the curvature perturbation evolves according to [143]

$\zeta^{\prime}(t, \mathbf{x})=-\frac{\delta p_{\mathrm{rel}}(t, \mathbf{x})}{\rho(t)+p(t)} H(t)$,

where a prime denotes differentiation with respect to the cosmic time. Here, $\rho(t)$ and $p(t)$ are the total energy and pressure densities, $H(t)$ the Hubble parameter, and $\delta p_{\text {rel }}(t, \mathbf{x})$ is the so-called nonadiabatic pressure density perturbation defined by $\delta p_{\text {rel }}(t, \mathbf{x})=\delta p(t, \mathbf{x})-\delta \rho(t, \mathbf{x}) p^{\prime} / \rho^{\prime}$, with $\delta p$ being the pressure density perturbation. Assuming that the electromagnetic field is just a small perturbation with respect to the background (which is dominated by the inflaton field), we can write $\rho=\rho_{\text {inf }}$ and $p=p_{\text {inf }}$. The evolution equation for the curvature perturbation introduced by the electromagnetic field, $\zeta^{\mathrm{em}}(t, \mathbf{x})$, is then

$\left(\zeta^{\mathrm{em}}\right)^{\prime}(t, \mathbf{x})=-\frac{\delta p_{\text {rel }, \mathrm{em}}(t, \mathbf{x})}{\rho_{\mathrm{inf}}(t)+p_{\text {inf }}(t)} H(t)$,

where $\delta p_{\text {rel,em }}(t, \mathbf{x})=\delta p_{\text {em }}(t, \mathbf{x})-\delta \rho_{\mathrm{em}}(t, \mathbf{x}) p_{\text {inf }}^{\prime} / \rho_{\text {inf }}^{\prime}$ is the nonadiabatic pressure perturbation due to the relative entropy perturbation between the electromagnetic and the inflaton fields. Here, $\delta \rho_{\mathrm{em}}$ and $\delta p_{\mathrm{em}}$ are the electromagnetic energy and pressure density perturbations, respectively, and they are the same quantities defined in Eq. (117), to wit, $\delta \rho_{\mathrm{em}}=\rho_{\mathrm{em}}$ and $\delta p_{\mathrm{em}}=p_{\mathrm{em}}$.
Assuming a quasi-de Sitter inflation characterized by the slow-roll parameter $\epsilon \ll 1, p_{\text {inf }}=(1-2 \epsilon / 3) \rho_{\text {inf }}$, and introducing the electromagnetic equation-of-state $w_{\mathrm{em}}=$ $\rho_{\mathrm{em}} / p_{\mathrm{em}}=\delta \rho_{\mathrm{em}} / \delta p_{\mathrm{em}}$, the solution of Eq. (124) reads

$\zeta^{\mathrm{em}}(t, \mathbf{x})=-3\left(1+w_{\mathrm{em}}\right) \frac{H}{2 \epsilon \rho_{\mathrm{inf}}} \int_{t_{i}}^{t} \mathrm{~d} t^{\prime} \delta \rho_{\mathrm{em}}\left(t^{\prime}, \mathbf{x}\right)$,

where $t_{i}$ is the time when electromagnetic fluctuations begin to develop, $\zeta^{\mathrm{em}}\left(t_{i}, \mathbf{x}\right)=0$, and

$w_{\mathrm{em}}=\frac{3 \xi_{1}-5 \xi_{2}}{3\left(3 \xi_{1}-\xi_{2}\right)}$.

In obtaining Eq. (125), we assumed, as in Ref. [143], that $H$, $\epsilon$, and $\rho_{\text {inf }}$ are constant during inflation, and that $p_{\text {inf }}^{\prime} \simeq \rho_{\text {inf }}^{\prime}$. Equation (126), instead, comes from Eqs. (98) and (99). In the case $w_{\mathrm{em}}=1 / 3$, we recover the result of Ref. [143]. The curvature perturbation in Eq. (125) is the key quantity from which observable quantities can be constructed and then compared to CMB results.

In Ref. [143], the standard kinetically coupled scenario for magnetogenesis was studied. In this case, the electromagnetic energy density is dominated by the electric part, so that, working in Fourier space, the electromagnetic energy spectrum can be approximated by $\delta \rho_{\mathrm{em}}(k, \eta)=\frac{1}{2} f(\phi) \mathcal{P}_{E}(k, \eta)$. The expression of the electric power spectrum (in the standard kinetically coupled scenario) can be obtained by using Eqs. (88) and (89), and taking $Z=1 / f(\phi)$ and $n=1$. It is $\mathcal{P}_{\mathrm{E}}(k, \eta)=\left(v^{2}\left|c_{\nu}\right|^{2} / \pi\right)(-k \eta)^{3+2 v} H^{4} / f(\phi)$. The scalinginvariant case corresponds to taking $v=-3 / 2$, and it gives $\mathcal{P}_{\mathrm{E}}(k, \eta)=\left(9 / 2 \pi^{2}\right) H^{4} / f(\phi)$. Accordingly, we have

$\delta \rho_{\mathrm{em}, *}(k, \eta)=\frac{9}{4 \pi^{2}} H^{4}$,

where, as below, a star indicates that the corresponding result is obtained in the standard kinetically coupled scenario for the case of a scaling-invariant electric power spectrum.

In our case, instead, the electric part does not make any contribution to the electromagnetic energy which is then dominated by the magnetic part (see discussion in Sect. 6). The expression for $\delta \rho_{\mathrm{em}}(k, \eta)$ is given in Eqs. (98), which we rewrite here for the sake of convenience,

$\delta \rho_{\mathrm{em}}(k, \eta)=\frac{9 n^{5} \Upsilon_{1}}{2 \pi^{2}} H^{4}$.

Comparing Eqs. (127) and (128), and taking into account Eq. (125), we see that the curvature perturbation in our case can be obtained by multiplying the result of Ref. [143] (for the scaling-invariant case) by a constant factor $\vartheta$,

$\zeta^{\mathrm{em}}=\vartheta\left(\xi_{1}, \xi_{2}\right) \zeta_{*}^{\mathrm{em}}$,

where

$\vartheta\left(\xi_{1}, \xi_{2}\right)=\frac{3\left(1+w_{\mathrm{em}}\right)}{2} n^{5} \Upsilon_{1}=n^{5}\left(2 \Upsilon_{1}-\Upsilon_{2}\right)$, 
and where, in the last equality of the above equation, we used Eqs. (100), (101), and (126).

\subsection{Scalar modes: spectrum, bispectrum, and trispectrum}

The observable quantities that can be constructed starting from the curvature perturbation $\zeta(t, \mathbf{x})$, are the corresponding $n$-points correlation functions in Fourier space. In particular, the actual sensitivity of CMB experiments allows us to put constraints on the 2-points correlator, the power spectrum of curvature perturbations, on the 3-point correlator, the bispectrum, and on the 4-points correlator, the trispectrum, defined via

$$
\begin{aligned}
\left\langle\zeta_{\mathbf{k}_{1}} \zeta_{\mathbf{k}_{2}}\right\rangle=(2 \pi)^{3} \delta\left(\mathbf{k}_{1}+\mathbf{k}_{2}\right) \frac{2 \pi^{2}}{k_{1}^{3}} \mathcal{P}_{\zeta} & \\
\left\langle\zeta_{\mathbf{k}_{1}} \zeta_{\mathbf{k}_{2}} \zeta_{\mathbf{k}_{3}}\right\rangle= & (2 \pi)^{3} \delta\left(\mathbf{k}_{1}+\mathbf{k}_{2}+\mathbf{k}_{3}\right)\left(2 \pi^{2} \mathcal{P}_{\zeta}\right)^{2} \\
& \times \frac{6}{5} f_{\mathrm{NL}}^{\text {local }} \frac{\sum_{i=1}^{3} k_{i}^{3}}{\prod_{i=1}^{3} k_{i}^{3}}, \\
\left\langle\zeta_{\mathbf{k}_{1}} \zeta_{\mathbf{k}_{2}} \zeta_{\mathbf{k}_{3}} \zeta \mathbf{k}_{4}\right\rangle= & (2 \pi)^{3} \delta\left(\mathbf{k}_{1}+\mathbf{k}_{2}+\mathbf{k}_{3}+\mathbf{k}_{4}\right)\left(2 \pi^{2} \mathcal{P}_{\zeta}\right)^{3} \\
& \times \tau_{\mathrm{NL}}\left[\frac{1}{\left(k_{1} k_{2} k_{13}\right)^{3}}+11 \text { permutations }\right]
\end{aligned}
$$

respectively, where a scaling-invariant power $\operatorname{spectrum} \mathcal{P}_{\zeta}(k)$ is assumed, and all quantities are evaluated at the end of inflation $\eta=\eta_{\text {end }}$. Here, $\zeta_{\mathbf{k}}$ is the Fourier-transformed curvature perturbation, $\langle\ldots\rangle$ indicates an ensemble average, $k_{i}=\left|\mathbf{k}_{i}\right|$, and $\mathbf{k}_{i j}=\left|\mathbf{k}_{i}+\mathbf{k}_{j}\right|$. The observable quantities, besides $\mathcal{P}_{\zeta}(k)$, are the local-type non-linearity (dimensionless) parameters $f_{\mathrm{NL}}^{\text {local }}$ and $\tau_{\mathrm{NL}}$, which parameterize the nonGaussian features of the primordial spectrum of curvature perturbations.

Let us observe that the 3-points correlator can be generally written as $\left\langle\zeta_{\mathbf{k}_{1}} \zeta_{\mathbf{k}_{2}} \zeta_{\mathbf{k}_{3}}\right\rangle=(2 \pi)^{3} \delta\left(\mathbf{k}_{1}+\mathbf{k}_{2}+\mathbf{k}_{3}\right) f_{\mathrm{NL}} \mathcal{P}_{\zeta}\left(k_{1}\right.$, $\left.k_{2}, k_{3}\right)$, where the bispectrum $\mathcal{P}_{\zeta}\left(k_{1}, k_{2}, k_{3}\right)$ measures the correlation among three perturbation modes [145]. The bispectrum can assume different forms which depend on the type of triangle formed by the three wave numbers $k_{1}, k_{2}$, and $k_{3}$. Local-type non-linearities are characterized by a bispectrum that is maximal for "squeezed" triangles with $k_{1} \ll k_{2} \simeq k_{3}$ (or permutations). Other types of configurations are possible, such as the equilateral and the orthogonal. They are, however, inessential for our discussion since a scaling-invariant electromagnetic field produces (under appropriate approximations [143]) only local-type shapes for the bispectrum and trispectrum.

Using the results of Ref. [143], appropriately re-scaled by using Eq. (129), we find for the electromagnetic part of the power spectrum of curvature perturbations, $\mathcal{P}_{\zeta}^{\mathrm{em}}$, and for the electromagnetic part of the local-type non-linearity parameters, $f_{\mathrm{NL}}^{\mathrm{em}}$ and $\tau_{\mathrm{NL}}^{\mathrm{em}}$,

$$
\begin{aligned}
& \mathcal{P}_{\zeta}^{\mathrm{em}}=192 \vartheta^{2} N_{\mathrm{cmb}}^{2} \Delta N\left(\mathcal{P}_{\zeta}^{\mathrm{inf}}\right)^{2}, \\
& f_{\mathrm{NL}}^{\mathrm{em}}=\frac{20}{3} \vartheta N_{\mathrm{cmb}} \frac{\mathcal{P}_{\zeta}^{\mathrm{em}}}{\mathcal{P}_{\zeta}^{\mathrm{inf}}}, \\
& \tau_{\mathrm{NL}}^{\mathrm{em}}=72 \vartheta^{2} N_{\mathrm{cmb}}^{2} \frac{\mathcal{P}_{\zeta}^{\mathrm{em}}}{\mathcal{P}_{\zeta}^{\text {inf }}},
\end{aligned}
$$

respectively. Here, it has been assumed that the dominant component of the power spectrum of curvature perturbations is generated by the inflaton,

$\mathcal{P}_{\zeta}^{\mathrm{em}} \ll \mathcal{P}_{\zeta}^{\mathrm{inf}}$,

where the power spectrum of curvature perturbations in slowroll inflation is [143]

$\mathcal{P}_{\zeta}^{\inf }=\frac{1}{24 \pi^{2} \epsilon}\left(\frac{M}{m_{\mathrm{Pl}}}\right)^{4}$.

Moreover, it has been assumed that $\Delta N>1$, where

$\Delta N=N_{\text {tot }}-N_{\mathrm{cmb}}$,

with $N_{\text {tot }}=-\ln \left|k_{\min } \eta_{\text {end }}\right|$ and $N_{\mathrm{cmb}}=-\ln \left|k_{\mathrm{cmb}} \eta_{\mathrm{end}}\right|$. Here, $k_{\min }$ is the wave numbers of the mode that crosses the horizon when magnetogenesis begins, and $k_{\mathrm{cmb}}$, referred to as the CMB scale, is the wave number of the largest scale CMB mode. We have assumed, in the previous sections, that magnetogenesis begins simultaneously with inflation, so that $k_{\min }=-1 / \eta_{i}$, and in turns $N_{\text {tot }}$ is the total number of $e$-fold of inflation. On the other hand, $N_{\mathrm{cmb}}$ is the number of $e$ folds between the moment at which the CMB mode leaves the horizon and the end of inflation. Assuming for simplicity an instantaneous reheating, we have [137]

$N_{\mathrm{cmb}} \simeq 61+\ln \frac{\lambda_{\mathrm{cmb}}}{4000 \mathrm{Mpc}}+\ln \frac{M}{10^{16} \mathrm{GeV}}$,

where $\lambda_{\mathrm{cmb}}=2 \pi / k_{\mathrm{cmb}}$. Taking $M=10^{16} \mathrm{GeV}$ and the CMB scale as large as the present Hubble radius, $\lambda_{\mathrm{cmb}}=$ $H_{0}^{-1} \simeq 4000 \mathrm{Mpc}$, we get $N_{\mathrm{cmb}} \simeq 61$.

We use the experimental results derived from Planck data [146-148],

$\mathcal{P}_{\zeta}^{\text {inf }}=2.2 \times 10^{-9}$ (best-fit),

$f_{\mathrm{NL}}^{\text {local }}=2.5 \pm 5.7$ (68\% C.L. $)$,

$\tau_{\mathrm{NL}}=(0.3 \pm 0.9) \times 10^{4}(68 \%$ C.L. $)$,

where $\mathcal{P}_{\zeta}^{\inf }(k)$ is evaluated at the pivot scale $k_{0}=0.05 \mathrm{Mpc}^{-1}$. Taking into account Eq. (141), we can conveniently rewrite Eqs. (134), (135), and (136) as

$$
\begin{aligned}
& \frac{\mathcal{P}_{\zeta}^{\mathrm{em}}}{\mathcal{P}_{\zeta}^{\text {inf }}} \simeq 1.6 \times 10^{-3} \vartheta^{2}\left(\frac{N_{\mathrm{cmb}}}{61}\right)^{2} \Delta N, \\
& f_{\mathrm{NL}}^{\mathrm{em}} \simeq 0.6 \vartheta^{3}\left(\frac{N_{\mathrm{cmb}}}{61}\right)^{3} \Delta N,
\end{aligned}
$$


$\tau_{\mathrm{NL}}^{\mathrm{em}} \simeq 0.4 \times 10^{3} \vartheta^{4}\left(\frac{N_{\mathrm{cmb}}}{61}\right)^{4} \Delta N$

respectively. Taking into account Eqs. (137), (141), (142), (144), and (145), we see that inflationary magnetogenesis is compatible with current $\mathrm{CMB}$ data if

$$
\begin{aligned}
|\vartheta| & \ll \frac{25}{\left(N_{\mathrm{cmb}} / 61\right)(\Delta N)^{1 / 2}}, \\
|\vartheta| & \lesssim \frac{2}{\left(N_{\mathrm{cmb}} / 61\right)(\Delta N)^{1 / 3}}, \\
|\vartheta| & \lesssim \frac{2}{\left(N_{\mathrm{cmb}} / 61\right)(\Delta N)^{1 / 4}},
\end{aligned}
$$

where the above three constraints come from the spectrum, bispectrum, and trispectrum of the curvature perturbation, respectively. Since $\Delta N$ is a quantity greater than unity, we obtain that the stringent constraint on $|\vartheta|$ comes either from the bispectrum if $\Delta N \lesssim(25 / 2)^{6} \simeq 4 \times 10^{6}$, or from the spectrum otherwise.

Let us analyze the three cases in Eq. (95), by assuming, for the sake of simplicity, that the stringent constraint on $|\vartheta|$ comes from the bispectrum.

(i) $\xi_{1}=0$. In this case (see Sect. 6), $\left\langle\left(T_{\mathrm{em}}\right)_{\nu}^{\mu}\right\rangle=0$, so that no curvature perturbations are generated by the inflationproduced electromagnetic field.

(ii) $\xi_{2}=0$. In this case (see Sect. 6.3), $n=1, \Upsilon_{1}=1$, and $\Upsilon_{2}=0$, which give $w_{\mathrm{em}}=1 / 3$ and $\vartheta=2$. For $\left(N_{\mathrm{cmb}} / 61\right)(\Delta N)^{1 / 3}$ of order unity, the case $\xi_{2}=0$ is then compatible with current $\mathrm{CMB}$ data on curvature perturbations, while for greater values it is excluded.

(iii) $\xi_{1} / \xi_{2} \in \mathbb{X}$. If $\left(N_{\mathrm{cmb}} / 61\right)(\Delta N)^{1 / 3}$ is of order unity, we have $n^{5}\left|2 \Upsilon_{1}-\Upsilon_{2}\right|=|\vartheta| \lesssim 2$. In this case, looking at Fig. 1 and remembering the discussion at the end of section VIc, we conclude that, with the exclusion of those values very close to the boundary $\partial \mathbb{X}=\{-1 / 3\} \cup\{1-2 / \sqrt{3}\} \cup\{1\} \cup\{1+2 / \sqrt{3}\}$, the inflation-produced electromagnetic field (whose magnetic component directly accounts for cosmic magnetic fields) generates curvature perturbations compatible with CMB data. The regions in the parameter space $\left(\xi_{1}, \xi_{2}\right)$ with acceptable curvature perturbations, instead, progressively shrink for increasing values of $\left(N_{\mathrm{cmb}} / 61\right)(\Delta N)^{1 / 3}$, as it appears from Fig. 3. Here, in the light gray regions the strength of the actual, scale-invariant magnetic field is in the range $10^{-13} \mathrm{G} \leq B_{0} \leq 5 \times 10^{-12} \mathrm{G}$ with no constraints on curvature perturbations imposed, while in the shrunk dark gray regions the constraint (149) has been imposed, [the darkness increases as $\left(N_{\mathrm{cmb}} / 61\right)(\Delta N)^{1 / 3}$ increases].

\subsection{Tensor modes}

The inflation-produced electromagnetic field affects, besides the scalar part of metric perturbation, also its tensor part, namely, it produces gravitational waves. However, we expect

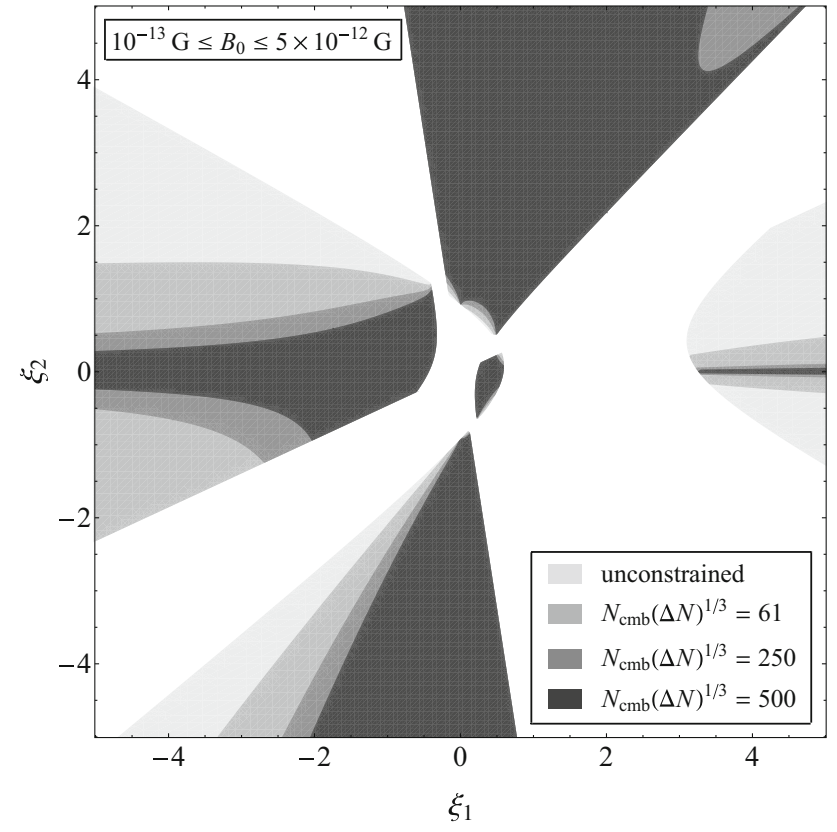

Fig. 3 Regions in the parameter space $\left(\xi_{1}, \xi_{2}\right)$, with $\xi_{1} / \xi_{2} \in \mathbb{X}$ [see Eq. (96)], where $10^{-13} \mathrm{G} \leq B_{0} \leq 5 \times 10^{-12} \mathrm{G}$ and the electromagnetic back-reaction on inflation is completely negligible, with no constraints on curvature perturbations imposed (light gray areas). The dark gray regions represent the same regions after imposing the constraint (148) which assures that scalar curvature perturbations generated by the inflation-produced electromagnetic field are compatible with CMB observations [the darkness increases as $\left(N_{\mathrm{cmb}} / 61\right)(\Delta N)^{1 / 3}$ increases]

that if $\mathcal{P}_{\zeta}^{\mathrm{em}} \ll \mathcal{P}_{\zeta}^{\mathrm{inf}}$, then the same it is true for the gravitational wave spectra, $\mathcal{P}_{\mathrm{GW}}^{\mathrm{em}} \ll \mathcal{P}_{\mathrm{GW}}^{\mathrm{inf}}$. This is because, as discussed in [80], the tensor modes are only produced gravitationally, while the dominant source of the scalar modes is the direct coupling between the inflaton and the electromagnetic field. In particular, the latter is enhanced with respect to the gravitational one by a factor inversely proportional to the slow-roll parameter. Therefore, we expect that

$\frac{\mathcal{P}_{\mathrm{GW}}^{\mathrm{em}}}{\mathcal{P}_{\mathrm{GW}}^{\mathrm{inf}}} \sim \epsilon \frac{\mathcal{P}_{\zeta}^{\mathrm{em}}}{\mathcal{P}_{\zeta}^{\text {inf }}}$

on physical grounds. Accordingly, due to the assumption (137), we can safely neglect the possible constraints on $\xi_{1}$ and $\xi_{2}$ coming from the production of gravitational waves in our model of inflationary magnetogenesis.

\section{Discussion}

So far, we have not distinguished between explicit (not dynamical) and spontaneous (dynamical) Lorentz violation. In the model at hand (described by a background rank- 2 tensor), the simplest realization of spontaneous Lorentz violation is realized when a dynamical tensor operator $\left(\mathcal{K}_{F}\right)_{\mu \nu}$ 
is coupled to the electromagnetic field and it acquires a vacuum expectation value different from zero, $\left(k_{F}\right)_{\mu \nu}=$ $\left\langle 0\left|\left(\mathcal{K}_{F}\right)_{\mu \nu}\right| 0\right\rangle$. In this case, the coupling between the rank-2 tensor field and the electromagnetic field would be described by Lagrangian $(25)$ with $\left(k_{F}\right)_{\mu \nu}$ replaced by $\left(\mathcal{K}_{F}\right)_{\mu \nu}$.

Decomposing $\left(\mathcal{K}_{F}\right)_{\mu \nu}$ in a classical part, $\left(k_{F}\right)_{\mu \nu}$, and a quantum part, $\delta\left(k_{F}\right)_{\mu \nu}$, to wit, writing

$\left(\mathcal{K}_{F}\right)_{\mu \nu}=\left(k_{F}\right)_{\mu \nu}+\delta\left(k_{F}\right)_{\mu \nu}$,

all the above analysis remains valid only if the quantum fluctuations $\delta\left(k_{F}\right)_{\mu \nu}$ are dynamically negligible. Assuming homogeneity and isotropy, we can write $\delta\left(k_{F}\right)_{v}^{\mu}=$ $\operatorname{diag}\left(\delta \rho_{K},-\delta p_{K},-\delta p_{K},-\delta p_{K}\right)$. All the arguments and results in Sect. 6 are then preserved if, roughly speaking,

$\left|\delta \rho_{K}\right|,\left|\delta p_{K}\right| \ll(-k \eta)^{2}\left(\frac{m_{\mathrm{Pl}}}{M}\right)^{4}$.

In this case, in fact, the contribution of the quantum fluctuations of $\left(\mathcal{K}_{F}\right)_{\mu \nu}$ to the electromagnetic energy-momentum tensor are negligible in the scaling-invariant case [see Eqs. (82)-(83) and Eqs. (90)-(91)]. Although the condition (152) seems to be very restrictive, it could be realized if, for example, $\delta\left(k_{F}\right)_{\nu}^{\mu} \sim(-k \eta)^{\alpha}$, with $\alpha \gtrsim 2$.

Finally, let us observe that the dynamics of the field $\left(\mathcal{K}_{F}\right)_{\mu \nu}$ is described by a Lagrangian of the type $\mathcal{L}_{\mathcal{K}}=$ $\mathcal{L}_{\mathcal{K} \text {, kin }}+\mathcal{L}_{\mathcal{K} \text {,int }}$, where $\mathcal{L}_{\mathcal{K} \text {, kin }}$ is the kinetic term (whose involved expression, for the case of de Sitter spacetime, can be found in [149]), while the interaction term $\mathcal{L}_{\mathcal{K}}$,int contains, besides self-coupling terms, all the couplings to other fields, included those with the photon and the inflaton. In a complete and self-consistent model, which is beyond the aim of this paper, one should also consider such a dynamics and consistently check that the field $\left(\mathcal{K}_{F}\right)_{\mu \nu}$ does not appreciably back-react on the dynamics of inflation and it generates curvature perturbations in agreement with $\mathrm{CMB}$ results.

\section{Conclusions}

Astrophysical observations definitely indicate the presence of microgauss magnetic fields in any type of galaxies, and they give compelling indications of the existence of strong, large-scale magnetic fields in galaxy clusters and cosmic voids.

A plausible hypothesis about their nature is that they have a primordial origin. In particular, presently observed magnetic fields could be nothing but primordial quantum electromagnetic fluctuations excited during an inflationary epoch of the universe which have survived until today.

However, since standard electrodynamics is conformally invariant, large-scale magnetic fields cannot be generated in a conformally invariant background spacetime, as a result of the Parker theorem. Accordingly, whatever is the mecha- nism responsible for generation of quantum electromagnetic fluctuations it must break conformal invariance of Maxwell theory.

In this paper, we have analyzed the generation of cosmic magnetic fields during (de-Sitter) inflation in a nonconformally invariant, Lorentz-violating effective model of electrodynamics. We have considered a Lorentz-violating extension of the kinetically coupled scenario for magnetogenesis, where the latter is described by a Lagrangian of the form $\mathcal{L}_{\mathrm{em}}=f(\phi) \mathcal{L}_{\mathrm{M}}$. Here, $\mathcal{L}_{\mathrm{M}}$ is the Maxwell Lagrangian and $f(\phi)$ is a generic coupling function between the photon and the inflaton $\phi$. Lorentz violation is introduced in our model by considering the Lagrangian $\mathcal{L}_{\mathrm{em}}=f(\phi)\left(\mathcal{L}_{\mathrm{M}}+\right.$ $\left.\mathcal{L}_{\mathrm{LV}}\right)$, where $\mathcal{L}_{\mathrm{LV}}$ incorporates all possible Lorentz-violating renormalizable operators.

We have restricted our analysis to the case where Lorentz symmetry breaking is implemented by the presence of a classical, homogeneous, and time-independent rank-2 symmetric background tensor. Working in the weak-coupling regime, we have shown that the creation of inflationary magnetic fields in this model proceeds similarly to the case of magnetogenesis in the standard kinetically coupled scenario. The key difference is that the new degrees of freedom represented by the components of the background tensor can be tuned in such a way to suppress the electric part of the inflation-produced electromagnetic energy-momentum tensor. This allows us to have, in de Sitter inflation with scale $\sim 10^{16} \mathrm{GeV}$, a self-consistent model of magnetogenesis where the inflation-produced electromagnetic field (i) does not appreciably back-react on inflation, (ii) it does not significantly affect the primordial spectrum of both scalar (Gaussian and non-Gaussian) and tensor curvature perturbations, and (iii) it evolves after inflation to give a strong, scalinginvariant magnetic field that directly accounts for galactic magnetic fields.

Open Access This article is distributed under the terms of the Creative Commons Attribution 4.0 International License (http://creativecomm ons.org/licenses/by/4.0/), which permits unrestricted use, distribution, and reproduction in any medium, provided you give appropriate credit to the original author(s) and the source, provide a link to the Creative Commons license, and indicate if changes were made.

Funded by $\mathrm{SCOAP}^{3}$.

\section{References}

1. P.P. Kronberg, Rep. Prog. Phys. 57, 325 (1994)

2. L.M. Widrow, Rev. Mod. Phys. 74, 775 (2002)

3. M. Giovannini, Int. J. Mod. Phys. D 13, 391 (2004)

4. J.P. Vallée, New Astron. Rev. 48, 763 (2004)

5. R. Beck, A.I.P. Conf. Proc. 1381, 117 (2011)

6. A. Kandus, K.E. Kunze, C.G. Tsagas, Phys. Rep. 505, 1 (2011)

7. D. Ryu, D.R.G. Schleicher, R.A. Treumann, C.G. Tsagas, L.M. Widrow, Space Sci. Rev. 166, 1 (2012). arXiv:1109.4055 [astroph.CO] 
8. L.M. Widrow, D. Ryu, D.R.G. Schleicher, K. Subramanian, C.G. Tsagas, R.A. Treumann, Space Sci. Rev. 166, 37 (2012). arXiv:1109.4052 [astro-ph.CO]

9. R. Durrer, A. Neronov, Astron. Astrophys. Rev. 21, 62 (2013). arXiv:1303.7121 [astro-ph.CO]

10. T. Prokopec, astro-ph/0106247 (unpublished)

11. R. Pakmor, F. Marinacci, V. Springel, Astrophys. J. 783, L20 (2014)

12. E. Fermi, Phys. Rev. 75, 1169 (1949)

13. N.D. Birrell, P.C.W. Davies, Quantum Fields in Curved Space (Cambridge University Press, New York, 1982)

14. L. Parker, D.J. Toms, Quantum Field Theory in Curved Spacetime: Quantized Fields and Gravity (Cambridge University Press, Cambridge, 2009)

15. M.S. Turner, L.M. Widrow, Phys. Rev. D 37, 2743 (1988)

16. B. Ratra, Astrophys. J. 391, L1 (1992)

17. F.D. Mazzitelli, F.M. Spedalieri, Phys. Rev. D 52, 6694 (1995)

18. D. Lemoine, M. Lemoine, Phys. Rev. D 52, 1955 (1995)

19. M. Gasperini, M. Giovannini, G. Veneziano, Phys. Rev. Lett. 75, $3796(1995)$

20. A.C. Davis, K. Dimopoulos, Phys. Rev. D 55, 7398 (1997)

21. M. Giovannini, M.E. Shaposhnikov, Phys. Rev. D 57, 2186 (1998)

22. A. Berera, T.W. Kephart, S.D. Wick, Phys. Rev. D 59, 043510 (1999)

23. M. Giovannini, Phys. Rev. D 62, 123505 (2000)

24. M. Giovannini, Phys. Rev. D 61, 087306 (2000)

25. M. Giovannini, Phys. Rev. D 64, 061301 (2001). arXiv:hep-ph/0104214

26. M. Giovannini, Phys. Lett. B 659, 661 (2008)

27. M. Giovannini, Phys. Rev. D 88, 083533 (2013)

28. A.L. Maroto, Phys. Rev. D 64, 083006 (2001)

29. K. Dimopoulos, astro-ph/0105488 (unpublished)

30. B.A. Bassett, G. Pollifrone, S. Tsujikawa, F. Viniegra, Phys. Rev. D 63, 103515 (2001)

31. K. Dimopoulos, T. Prokopec, O. Tornkvist, A.C. Davis, Phys. Rev. D 65, 063505 (2002)

32. M. Marklund, P.K.S. Dunsby, M. Servin, G. Betschart, C. Tsagas, Class. Quant. Grav. 20, 1823 (2003)

33. K. Bamba, J. Yokoyama, Phys. Rev. D 69, 043507 (2004)

34. G. Betschart, P.K.S. Dunsby, M. Marklund, Class. Quantum Grav. 21, 2115 (2004)

35. T. Prokopec, E. Puchwein, Phys. Rev. D 70, 043004 (2004)

36. A. Ashoorioon, R.B. Mann, Phys. Rev. D 71, 103509 (2005)

37. C.G. Tsagas, Phys. Rev. D 72, 123509 (2005)

38. C.G. Tsagas, Class. Quantum Grav. 22, 393 (2005)

39. M.R. Garousi, M. Sami, S. Tsujikawa, Phys. Lett. B 606, 1 (2005)

40. C.G. Tsagas, A. Kandus, Phys. Rev. D 71, 123506 (2005)

41. M.M. Anber, L. Sorbo, JCAP 0610, 018 (2006)

42. C. Zunckel, G. Betschart, P.K.S. Dunsby, M. Marklund, Phys. Rev. D 73, 103509 (2006)

43. K. Bamba, M. Sasaki, JCAP 0702, 030 (2007)

44. A. Akhtari-Zavareh, A. Hojati, B. Mirza, Prog. Theor. Phys. 117, 803 (2007)

45. K.E. Kunze, Phys. Lett. B 623, 1 (2005)

46. K.E. Kunze, Phys. Rev. D 77, 023530 (2008)

47. L. Campanelli, P. Cea, G.L. Fogli, L. Tedesco, Phys. Rev. D 77, 043001 (2008)

48. L. Campanelli, P. Cea, G.L. Fogli, L. Tedesco, Phys. Rev. D 77, $123002(2008)$

49. K. Bamba, JCAP 0710, 015 (2007)

50. K. Bamba, S.D. Odintsov, JCAP 0804, 024 (2008)

51. K. Bamba, N. Ohta, S. Tsujikawa, Phys. Rev. D 78, 043524 (2008)

52. K. Bamba, S. Nojiri, S.D. Odintsov, Phys. Rev. D 77, 123532 (2008)

53. K. Bamba, C.Q. Geng, S.H. Ho, JCAP 0811, 013 (2008)

54. L. Campanelli, Int. J. Mod. Phys. D 18, 1395 (2009)
55. R. Durrer, L. Hollenstein, R.K. Jain, JCAP 1103, 037 (2011)

56. C.T. Byrnes, L. Hollenstein, R.K. Jain, F.R. Urban, JCAP 1203, 009 (2012)

57. J. Beltran Jimenez, A.L. Maroto, Phys. Rev. D 83, 023514 (2011) (see also D.N. Vollick, Phys. Rev. D 86, 084057, 2012)

58. S. L. Cheng, W. Lee, K.W. Ng, arXiv:1409.2656 [astro-ph.CO]

59. K. Bamba, Phys. Rev. D 91, 043509 (2015)

60. A. Dolgov, Phys. Rev. D 48, 2499 (1993)

61. J.D. Barrow, C.G. Tsagas, Phys. Rev. D 77, 107302 (2008)

62. J.D. Barrow, C.G. Tsagas, Erratum-ibid. D 77, 109904 (2008)

63. J.D. Barrow, C.G. Tsagas, Mon. Not. R. Astron. Soc. 414, 512 (2011)

64. J.D. Barrow, C.G. Tsagas, K. Yamamoto, Phys. Rev. D 86, 023533 (2012)

65. Y. Shtanov, V. Sahni, JCAP 1201, 088 (2013)

66. C.G. Tsagas, arXiv:1412.4806 [astro-ph.CO]

67. L. Campanelli, Phys. Rev. Lett. 111, 061301 (2013)

68. R. Durrer, G. Marozzi, M. Rinaldi, Phys. Rev. Lett. 111, 229001 (2013)

69. L. Campanelli, Phys. Rev. Lett. 111, 229002 (2013)

70. P.G. Tinyakov, F.R. Urban, arXiv:1309.2270 [astro-ph.CO]

71. I. Agullo, J. Navarro-Salas, arXiv:1309.3435 [gr-qc]

72. I. Agullo, J. Navarro-Salas, A. Landete, Phys. Rev. D 90, 124067 (2014)

73. V. Demozzi, V. Mukhanov, H. Rubinstein, JCAP 0908, 025 (2009)

74. F.A. Membiela, Nucl. Phys. B 885, 196 (2014)

75. R.J.Z. Ferreira, R.K. Jain, M.S. Sloth, JCAP 1310, 004 (2013)

76. R.J.Z. Ferreira, R.K. Jain, M.S. Sloth, JCAP 1406, 053 (2014)

77. C. Caprini, L. Sorbo, JCAP 1410, 056 (2014)

78. G. Tasinato, JCAP 1503, 040 (2015)

79. R.Z. Ferreira, J. Ganc, arXiv:1411.5362 [astro-ph.CO]

80. N. Barnaby, R. Namba, M. Peloso, Phys. Rev. D 85, 123523 (2012)

81. R. Gambini, J. Pullin, Phys. Rev. D 59, 124021 (1999)

82. V.A. Kostelecky, S. Samuel, Phys. Rev. D 39, 683 (1989)

83. K. Dolag, M. Bartelmann, H. Lesch, Astron. Astrophys. 387, 383 (2002)

84. K. Dolag, private communication

85. B. Cheng, A.V. Olinto, D.N. Schramm, J.W. Truran, Phys. Rev. D 54, 4714 (1996)

86. L. Campanelli, Phys. Rev. D 84, 123521 (2011)

87. T. Kahniashvili, A.G. Tevzadze, S.K. Sethi, K. Pandey, B. Ratra, Phys. Rev. D 82, 083005 (2010)

88. T. Kahniashvili, Y. Maravin, A. Natarajan, N. Battaglia, A.G. Tevzadze, Astrophys. J. 770, 47 (2013)

89. M. Giovannini, Class. Quantum Grav. 23, R1 (2006)

90. P.A.R. Ade et al., (Planck Collaboration), arXiv:1502.01594 [astro-ph.CO]

91. L. Campanelli, P. Cea, L. Tedesco, Phys. Rev. Lett. 97, 131302 (2006)

92. L. Campanelli, P. Cea, L. Tedesco, Phys. Rev. D 76, 063007 (2007)

93. T. Kahniashvili, G. Lavrelashvili, B. Ratra, Phys. Rev. D 78, 063012 (2008)

94. J. Chluba, D. Paoletti, F. Finelli, J.A. Rubino-Martin, arXiv:1503.04827 [astro-ph.CO]

95. P.P. Kronberg, P. Simard-Normandin, Nature 263, 653 (1976)

96. P.P. Kronberg, A.I.P. Conf. Proc. 558, 451 (2001)

97. A. Neronov, I. Vovk, Science 328, 73 (2010)

98. F. Tavecchio, G. Ghisellini, L. Foschini, G. Bonnoli, G. Ghirlanda, P. Coppi, Mon. Not. R. Astron. Soc. 406, L70 (2010)

99. F. Tavecchio, G. Ghisellini, G. Bonnoli, L. Foschini, Mon. Not. R. Astron. Soc. 414, 3566 (2011)

100. J. Martin, J. Yokoyama, JCAP 0801, 025 (2008)

101. P.A.R. Ade et al., BICEP2 Collaboration, Phys. Rev. Lett. 112, 241101 (2014)

102. V.A. Kostelecký, R. Potting, S. Samuel, in Proceedings of the 1991 Joint International Lepton-Photon Symposium and Europhysics 
Conference on High Energy Physics, ed. by S. Hegarty, K. Potter,

E. Quercigh (World Scientific, Singapore, 1992)

103. O. Bertolami, D.F. Mota, Phys. Lett. B 455, 96 (1999)

104. A. Mazumdar, M.M. Sheikh-Jabbari, Phys. Rev. Lett. 87, 011301 (2001)

105. J. Gamboa, J. Lopez-Sarrion, Phys. Rev. D 71, 067702 (2005)

106. K. Bamba, J. Yokoyama, 70, 083508 (2004)

107. L. Campanelli, P. Cea, G.L. Fogli, Phys. Lett. B 680, 125 (2009)

108. L. Campanelli, P. Cea, Phys. Lett. B 675, 155 (2009)

109. L. Campanelli, Phys. Rev. D 80, 063006 (2009)

110. L. Campanelli, Phys. Rev. D 90, 105014 (2014)

111. A.P. Kouretsis, Eur. Phys. J. C 74, 2879 (2014)

112. V.A. Kostelecky, Phys. Rev. D 69, 105009 (2004)

113. D. Colladay, V.A. Kostelecky, Phys. Rev. D 58, 116002 (1998)

114. W.E. Boyce, R.C. DiPrima, Elementary Differential Equations and Boundary Value Problems (Wiley, New York, 1986)

115. V.A. Kostelecky, N. Russell, Rev. Mod. Phys. 83, 11 (2011)

116. L. Campanelli, Eur. Phys. J. C 74, 2690 (2014)

117. A. Brandenburg, K. Enqvist, P. Olesen, Phys. Rev. D 54, 1291 (1996)

118. P. Olesen, Phys. Lett. B 398, 321 (1997)

119. D.T. Son, Phys. Rev. D 59, 063008 (1999)

120. D. Biskamp, W.C. Müller, Phys. Rev. Lett. 83, 2195 (1999)

121. M. Christensson, M. Hindmarsh, A. Brandenburg, Phys. Rev. E 64, 056405 (2001)

122. M. Christensson, M. Hindmarsh, A. Brandenburg, Astron. Nachr. 326, 393 (2005). arXiv:astro-ph/0209119

123. A. Brandenburg, Science 292, 2440 (2001)

124. R. Banerjee, K. Jedamzik, Phys. Rev. Lett. 91, 251301 (2003)

125. R. Banerjee, K. Jedamzik, Erratum-ibid. 93, 179901 (2004)

126. R. Banerjee, K. Jedamzik, Phys. Rev. D 70, 123003 (2004)

127. L. Campanelli, Phys. Rev. D 70, 083009 (2004)

128. L. Campanelli, Phys. Rev. Lett. 98, 251302 (2007)

129. T. Kahniashvili, A. Brandenburg, A.G. Tevzadze, B. Ratra, Phys. Rev. D 81, 123002(2010)
130. A.G. Tevzadze, L. Kisslinger, A. Brandenburg, T. Kahniashvili, Astrophys. J. 759, 54 (2012)

131. T. Kahniashvili, A. G. Tevzadze, A. Brandenburg, A. Neronov, arXiv:1212.0596 [astro-ph.CO]

132. M. Giovannini, Phys. Rev. D 85, 043006 (2012)

133. A. Berera, M. Linkmann, Phys. Rev. E 90, 041003 (2014)

134. A. Brandenburg, T. Kahniashvili, A.G. Tevzadze, Phys. Rev. Lett. 114(7), 075001 (2015)

135. T. Kahniashvili, A. Brandenburg, L. Campanelli, B. Ratra, A.G. Tevzadze, Phys. Rev. D 86, 103005 (2012)

136. K. Dimopoulos, A.C. Davis, Phys. Lett. B 390, 87 (1997)

137. E.W. Kolb, M.S. Turner, The Early Universe (Addison-Wesley, Redwood City, 1990)

138. K. A. Olive, CERN Yellow Report CERN-2010-002, pp. 149-196. arXiv:1005.3955 [hep-ph]

139. S.W. Hawking, G.F.R. Ellis, The Large Scale Structure of SpaceTime (Cambridge University Press, Cambridge, 1973)

140. L.D. Landau, E.M. Lifshitz, The Classical Theory of Fields (Pergamon Press, Oxford, 1971)

141. T. Suyama, J. Yokoyama, Phys. Rev. D 86, 023512 (2012)

142. M. Shiraishi, E. Komatsu, M. Peloso, N. Barnaby, JCAP 1305, 002 (2013)

143. T. Fujita, S. Yokoyama, JCAP 1309, 009 (2013)

144. D.H. Lyth, K.A. Malik, M. Sasaki, JCAP 0505, 004 (2005)

145. P.A.R. Ade et al., (Planck Collaboration), Astron. Astrophys. 571, A24 (2014)

146. P.A.R. Ade et al., (Planck Collaboration), Astron. Astrophys. 571, A16 (2014)

147. P. A. R. Ade et al., (Planck Collaboration), arXiv:1502.01592 [astro-ph.CO]

148. C. Feng, A. Cooray, J. Smidt, J. O’Bryan, B. Keating, D. Regan, arXiv: 1502.00585 [astro-ph.CO]

149. C. Fronsdal, Phys. Rev. D 20, 848 (1979) 